\title{
Clonal tracing reveals diverse patterns of response to immune checkpoint blockade
}

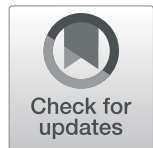

Shengqing Stan $\mathrm{Gu}^{1,2,3+}$, Xiaoqing Wang ${ }^{3,4,5+}$, Xihao Hu${ }^{1,2,3 \dagger}$, Peng Jiang ${ }^{1,2,3}$, Ziyi $\mathrm{Li}^{1,6}$, Nicole Traugh ${ }^{1,2}$, Xia Bu $\mathrm{Bu}^{4,5}$, Qin Tang ${ }^{3,4,5}$, Chenfei Wang ${ }^{1,2,3}$, Zexian Zeng ${ }^{1,2,3}$, Jingxin Fu ${ }^{1,6}$, Cliff Meyer ${ }^{1,2,3}$, Yi Zhang $^{1,2,3}$, Paloma Cejas $s^{3,4}$,

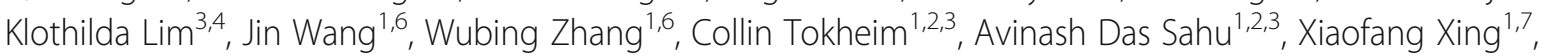
Benjamin Kroger ${ }^{8}$, Zhangyi Ouyang ${ }^{1}$, Henry Long ${ }^{3,4}$, Gordon J. Freeman ${ }^{4,5^{*}+}$, Myles Brown ${ }^{3,4,5^{*} \dagger}$ and X. Shirley Liu ${ }^{1,2,3^{*}+}$ (iD

\author{
* Correspondence: \\ gordon_freeman@dfci.harvard.edu; \\ myles_brown@dfci.harvard.edu; \\ xsliu@ds.dfci.harvard.edu \\ †Shengqing Stan Gu, Xiaoqing \\ Wang, and Xihao Hu contributed \\ equally to this work. \\ ${ }^{\dagger}$ Gordon J. Freeman, Myles Brown, \\ and $X$. Shirley Liu are senior authors. \\ ${ }^{4}$ Department of Medical Oncology, \\ Dana-Farber Cancer Institute, \\ Boston, MA 02215, USA \\ ${ }^{3}$ Center for Functional Cancer \\ Epigenetics, Dana-Farber Cancer \\ Institute, Boston, MA 02215, USA \\ 'Department of Data Science, \\ Dana-Farber Cancer Institute, \\ Boston, MA 02215, USA \\ Full list of author information is \\ available at the end of the article
}

\section{$\triangle B M C$}

\section{Background}

Immune checkpoint blockade (ICB) therapy, primarily anti-PD-1/PD-L1 and antiCTLA-4, has been shown to induce remarkable response across different cancers in

(c) The Author(s). 2020 Open Access This article is licensed under a Creative Commons Attribution 4.0 International License, which permits use, sharing, adaptation, distribution and reproduction in any medium or format, as long as you give appropriate credit to the original author(s) and the source, provide a link to the Creative Commons licence, and indicate if changes were made. The images or other third party material in this article are included in the article's Creative Commons licence, unless indicated otherwise in a credit line to the material. If material is not included in the article's Creative Commons licence and your intended use is not permitted by statutory regulation or exceeds the permitted use, you will need to obtain permission directly from the copyright holder. To view a copy of this licence, visit http://creativecommons.org/licenses/by/4.0/. The Creative Commons Public Domain Dedication waiver (http://creativecommons.org/publicdomain/zero/1.0/) applies to the data made available in this article, unless otherwise stated in a credit line to the data.

Keywords: Clonal tracing, Immune checkpoint blockade, Heterogeneity, Tumor microenvironment, Mathematical modeling 
multiple clinical cohorts [1-8]. However, only a minority of patients respond to ICB, and acquired resistance can occur in some early responders [9]. The mechanisms of resistance have been of extensive interest. On the one hand, multiple cancer cell-intrinsic mechanisms have been identified, including lack of a high neoantigen load [2, 3, 10, 11], impaired response or prolonged exposure to IFN $\gamma$ [12-15], defects in antigen presentation [16, 17], activation of certain oncogenic pathways/signatures [18-20], and other mechanisms [20, 21]. On the other hand, multiple cancer cell-extrinsic mechanisms have been reported to modulate ICB response, involving microenvironmental T cells [22-27], B cells [28-30], myeloid cells [31-33], natural killer cells [34], cancer-associated fibroblasts [34, 35], and gut microbiota [36-38]. The simultaneous contributions of both cancer cell-intrinsic and cancer cellextrinsic factors present a challenge in prediction of ICB response for each tumor.

As the major mechanism of action for ICB is thought to be through the stimulation of $\mathrm{T}$ cell cytotoxicity in the tumors, two distinct models of tumor immune evasion have been proposed: the induction of $\mathrm{T}$ cell dysfunction in tumors with high infiltration of cytotoxic $\mathrm{T}$ lymphocytes (CTLs) and the suppression of $\mathrm{T}$ cell infiltration in tumors with low CTL levels $[39,40]$. Our group previously developed a computational method, TIDE, which comprehensively evaluates $\mathrm{T}$ cell dysfunction and $\mathrm{T}$ cell exclusion signatures from the pre-treatment tumor gene expression data to predict patient responses to ICB treatment [41]. TIDE demonstrated promising performance in predicting patient responses based on published ICB clinical trials and also revealed new candidate ICBresistance regulators. However, the performance of TIDE still varies across different clinical cohorts.

Tumors of different tissue origins and genetic backgrounds have different prognoses in response to ICB $[9,42]$, suggesting that different gene expression and/or mutation profiles can have a strong influence on ICB response. However, it remains unclear whether tumors with a defined genetic background have a stereotypic or heterogeneous response to ICB. Answering this question is largely limited by a lack of experimental models. No two naturally occurring tumors are identical, making it difficult to study this question directly in human tumors. Patient-derived xenograft (PDX) models suffer from a lack of adaptive immune system in the recipient mice, precluding the assessment of ICB response. Genetically engineered mouse (GEM) models offer an intact immune system, but usually lack a sufficient mutational burden to elicit an effective immune response. Several syngeneic transplantation models harbor sufficient mutation loads and show response to ICB treatment in vivo, providing a powerful tool to study this question. However, thus far, no studies have assessed the heterogeneity of clonal evolution in response to ICB.

Clonal tracing is a powerful technique that can track and quantify the abundance of different clonal lineages with remarkable sensitivity and reproducibility. It has been used to interrogate a variety of biological processes that involve cellular heterogeneity, including stem/progenitor cell maintenance/differentiation [43-45], T cell recruitment/ expansion [46, 47], cancer clonal evolution [48], tumor-initiating potential [49], metastatic potential [50], and response to targeted therapies [51,52]. In this study, we utilized clonal tracing and mathematical modeling to dissect cancer clonal constitution in response to ICB treatment. We performed DNA barcoding of mouse cancer cells to label each cell and its progeny with a unique barcode and examined the clonal constitution of cancer cells under ICB treatment in different hosts. To interrogate the cancer 
cell-intrinsic contribution to ICB resistance, we compared the frequency of each clone in different treatment groups. To interrogate the cancer cell-extrinsic contribution to ICB resistance, we compared the clonal constitution of each tumor harvested from different mice with the same ICB treatment. Based on these comparisons, we further evaluated the cancer cell-intrinsic and cancer cell-extrinsic signatures of immune evasion in response to ICB treatment.

\section{Results}

Performance of biomarkers for ICB response is inconsistent across clinical trials

Multiple clinical studies have been performed to address the efficacy of ICB in multiple cancer types and investigate the biomarkers of response for optimal stratification of patients for treatment. Pre-treatment PD-L1 level was proposed as a biomarker for better response to nivolumab, an anti-PD-1 antibody [1]. Higher tumor mutation burden and DNA mismatch repair deficiency were also identified to correlate with better ICB response $[2,3]$. We performed a closer assessment of such biomarkers in recent clinical cohorts, which revealed inconsistent performance in segregating ICB responders and non-responders (Fig. 1a, b). For example, higher CD274 mRNA level significantly correlates with better ICB response in the Gide et al. study [53], but not in multiple other studies that used anti-PD-1 or anti-PD-L1 [20, 35, 54, 55] (Fig. 1a). Consistent with this, a literature search also revealed variable prediction power of PD-L1 protein level on anti-PD-1 response in different studies $[3,56]$. Similarly, higher tumor mutation load significantly correlates with better response in the Mariathasan et al. study [35], but not in multiple other studies [20,55] (Fig. 1b). We systematically evaluated other frequently reported biomarkers in existing clinical cohorts, including microsatellite instability (MSI) score, CD8a/CD8b, IFNG, and intra-tumoral T cell or B cell clonality. All of these biomarkers showed highly variable performance in predicting the likelihood of response to ICB (Fig. 1c).

There are two complementary models that can explain such inconsistent performance (Fig. 1d). In the first model, the different genetic mutation profiles and epigenetic status of cancer cells between different tumors determine the different responses to immunotherapy. According to this model, each clinical cohort samples a small number of patients out of the vast number of potential combinations of mutation profiles and epigenetic status, and the biomarkers correlated with response in a certain trial occur by chance, with the observations not repeated in other trials. This model is substantiated by studies identifying different cancer cell-intrinsic mechanisms of immune resistance $[12,57]$ and indicates that larger sample sizes are needed to achieve reliable biomarker performance. The second model assumes that even for the same cancer cells grown in different patients, the response can still be diverse due to tumor microenvironment or other host factors. If the second model is correct, a combination of biomarkers measuring orthogonal aspects of the tumor would be required for a reliable prediction of response. Although both models may hold true, with each partially explaining the response to ICB in any given patient, the second model is more difficult to test in human clinical studies because every naturally occurring human tumor is genetically and contextually distinct. We therefore established a novel mouse system to address this problem. 


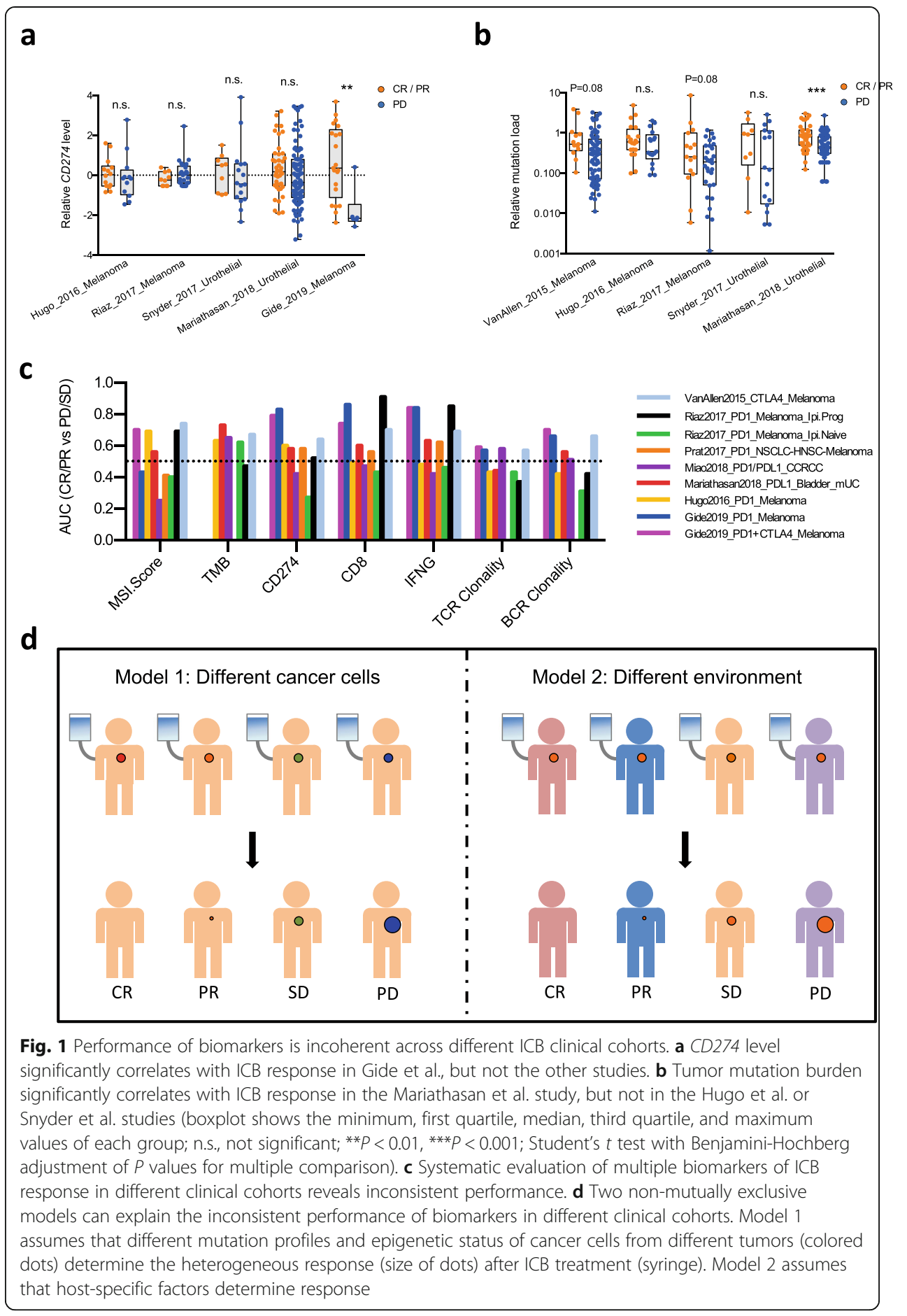

\section{Establishing an in vivo system to assess the heterogeneity in ICB response}

To study the response patterns to ICB treatment, we first used the mouse CT26 colorectal cancer cell line model, which shows moderate response to anti-PD1 or antiCTLA4 treatment with remarkable heterogeneity [58]. We used the ClonTracer barcoding system [51] to uniquely label clones in CT26 cell populations for in vivo study. We implemented several stringent criteria during transduction to ensure unique labeling of each transduced cell with a distinct barcode. First, we used a much lower 
number of CT26 cells for transduction compared to the total complexity of the ClonTracer barcode library, which minimizes the chance of labeling different cells with the same barcode. Second, we performed transduction at very low multiplicity of infection $(\mathrm{MOI}=0.01)$, which lowers the chance of infecting one cell with multiple virions. After transduction, CT26 cells carrying barcodes were selected and expanded in vitro, and high-throughput sequencing was performed to evaluate barcode representation in the population of cells. We found that barcoded cells from the same transduced batch showed good consistency in barcode identity and frequency, whereas those from different batches showed minimal overlap (Additional file 1: Fig. S1), indicating good reproducibility and uniqueness of our barcode approach. To guarantee sufficient coverage of each barcoded cancer clone for in vivo experiments, we selected the batch of transduced cells with $\sim 2000$ distinct barcodes for further study.

We transplanted 250,000 in vitro expanded barcoded cells per site into syngeneic recipient Balb/c mice subcutaneously (2 sites per mouse) and allowed the tumors to establish in vivo for 7 days. We then treated the recipient mice with control IgG, antiPD-1, or anti-CTLA-4, and observed significantly reduced tumor growth upon ICB treatment (Fig. 2a, b). ICB treatment significantly increased the intra-group variance in tumor size (Fig. 2c), indicating individual heterogeneity in ICB response. In addition, we examined this finding in multiple other syngeneic transplantation models (MC38, 4T1, and EMT6) and found consistently that ICB treatment led to higher variance in tumor size in each model (Additional file 1: Fig. S2a-c).

To evaluate the barcode constitution in each tumor, we PCR-amplified and sequenced the DNA barcodes from each remaining tumor and from cells cultured in vitro. Compared to the in vitro samples, over $80 \%$ of clones were consistently absent in the in vivo samples, suggesting strong engraftment selection (Additional file 1: Fig. S2d-e). The in vivo barcodes showed significantly higher clonality in the anti-PD-1- or anti-CTLA-4-treated tumors compared to control IgG-treated tumors, indicating clonal selection by ICB treatment (Fig. 2d). The clones enriched in the anti-PD-1 treatment also tended to be enriched in anti-CTLA-4 treatment, suggesting shared cancer cellintrinsic ICB-resistance mechanisms for the two regimens (Fig. 2e).

Our observations on CT26 syngeneic tumors treated with anti-PD-1 or anti-CTLA-4 suggest heterogeneous response at both the bulk tumor level and at the cancer clone level. At the bulk tumor level, different tumors initiated by the same clones of cancer cells exhibit diverse growth dynamics in response to ICB. At the cancer clone level, different clones within the same tumor show heterogeneous sensitivity to ICB treatment. Based on these results, we further examined whether the bulk tumor-level heterogeneity is associated with clonal level heterogeneity.

\section{ICB responders had stronger clonal selection and higher immune infiltration than non- responders}

The finding that different tumors composed of the same cancer clones responded differently to ICB treatment indicates a cancer-extrinsic mechanism of response from the host. Indeed, comparing the tumors that developed on the left and right flanks on each recipient mouse, we found their tumor size to be significantly positively correlated, especially in the anti-PD-1- and anti-CTLA-4-treated groups (Fig. 3a). In addition, 


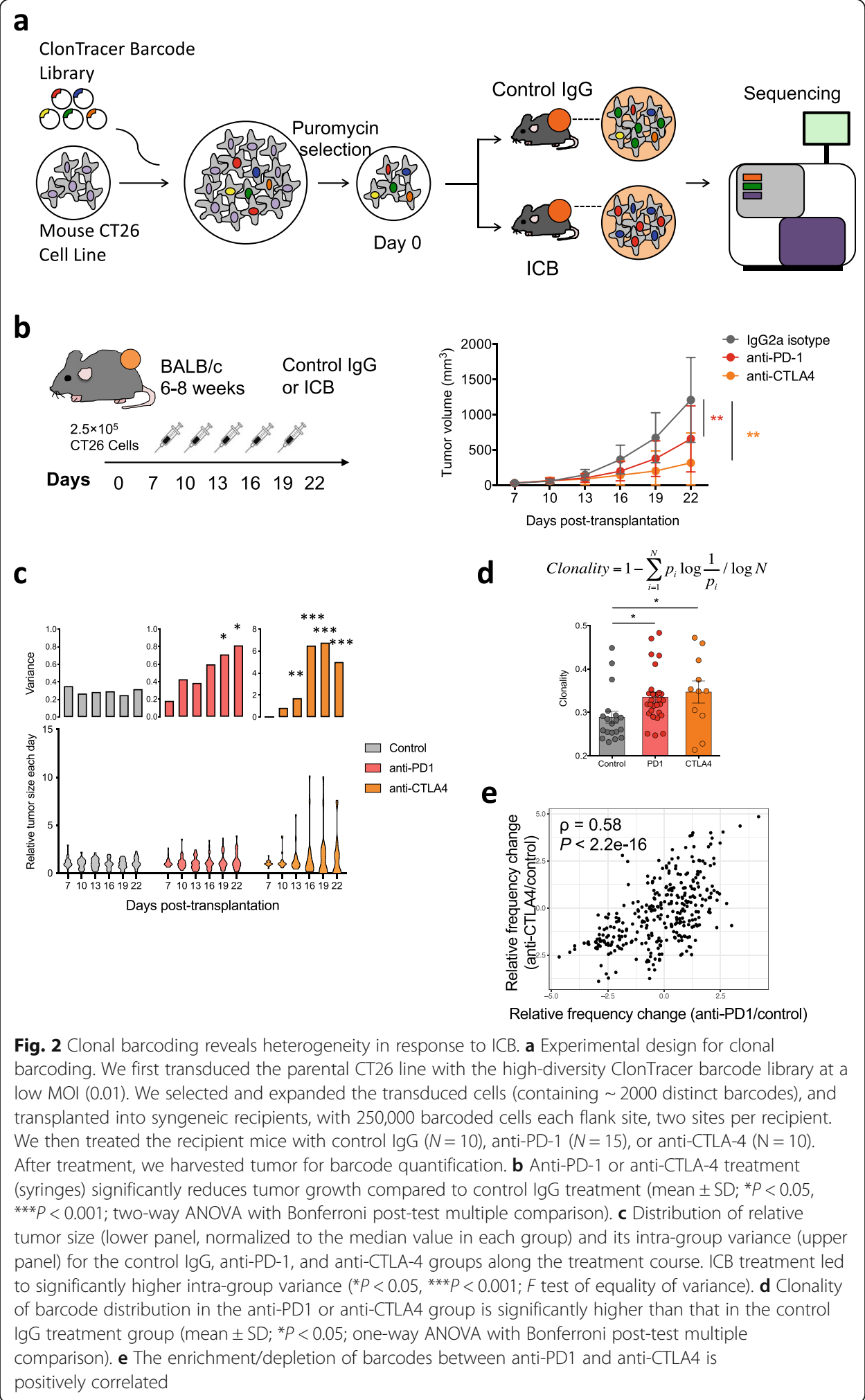

barcode distribution in the two tumors from the same mouse showed significantly higher correlation than those in tumors from different mice (Fig. 3b). These results 


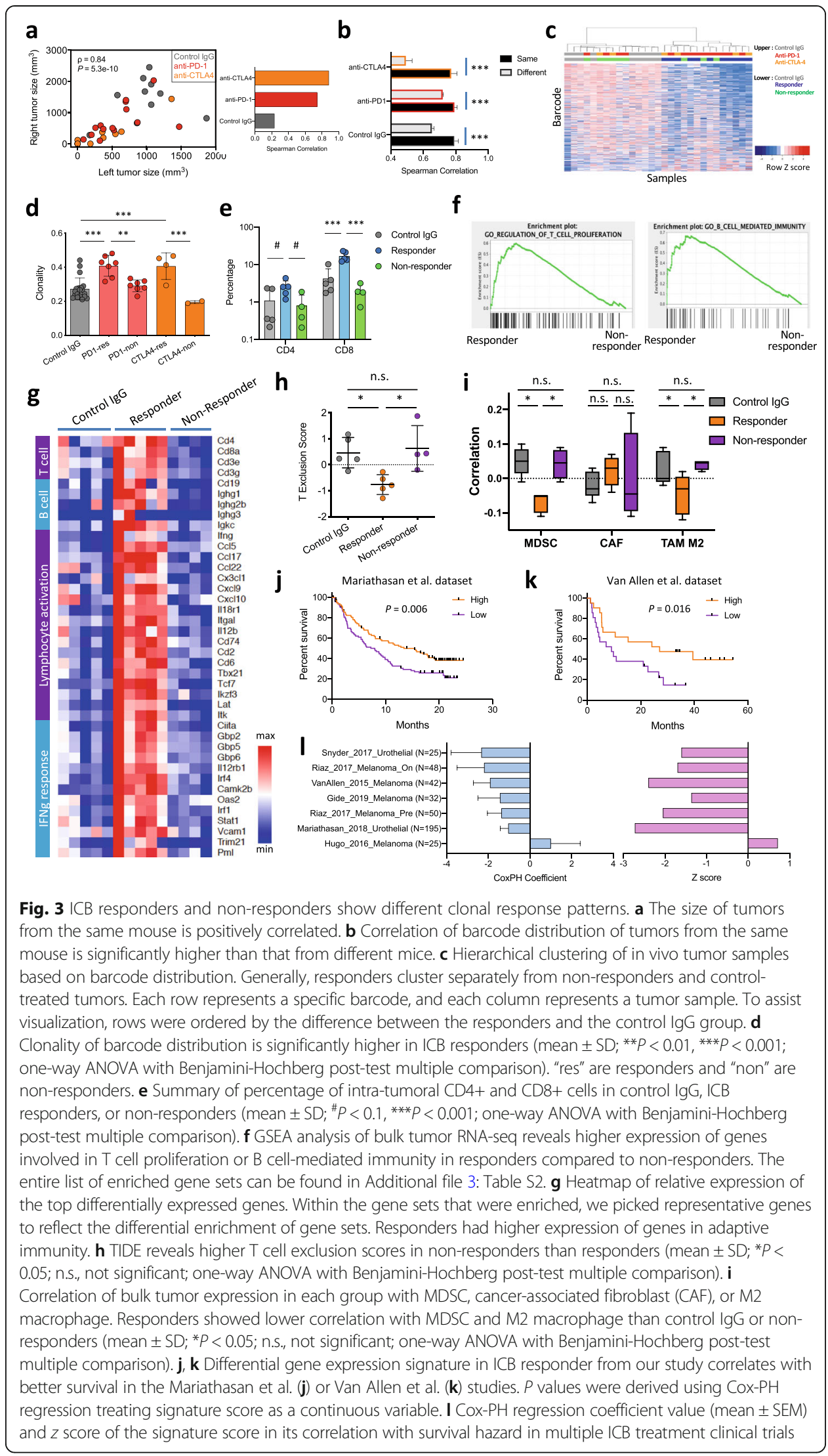


indicate that systemic factors at the host level may influence tumor growth in both control IgG- and ICB-treated groups.

To investigate why different ICB responses arose between tumors derived from the same batch of cancer cells, we identified ICB responders and non-responders by tumor size post-treatment (Additional file 1: Fig. S3a-b) and compared their clonal distributions and tumor microenvironments. Hierarchical clustering and a Kolmogorov-Smirnov test based on barcode distribution showed that the ICB responders had distinct clonal constitution of cancer cells compared to control IgG-treated mice (Fig. 3c, Additional file 1: Fig. S3c). In addition, the ICB responders had higher clonality of barcode distribution, in other words, higher unevenness in barcode distribution, than the non-responders (Fig. 3d, Additional file 1: Fig. S3d). Further examination of the tumor microenvironment by IHC revealed that the responders had higher infiltration of $\mathrm{T}$ cells in the tumors (Fig. 3e, Additional file 1: Fig. S3e). This prompted us to further compare the responders and the non-responders by bulk tumor RNA-seq. Principal component analysis (PCA) revealed that the ICB responders had distinct gene expression from the non-responders (Additional file 1: Fig. S3f). Consistent with observed higher immune infiltration from IHC, the responder tumors had higher expression of genes involved in antigen binding, adaptive immune response, and lymphocyte-mediated immunity than the non-responders (Fig. 3f, g, Additional file 1: Fig. S3g), and lower expression of genes involved in protein translation and oxidative phosphorylation (Additional file 1: Fig. S3h). The responders also showed higher expression of pro-inflammatory cytokines/chemokines, such as Ifng, Cxcl9, Cxcl10, and Ccl5 (Fig. 3g). Furthermore, we utilized another mouse model (MC38 colorectal cancer) to investigate the difference between ICB responders and nonresponders. Again, we observed strong association between primary response to ICB and cancer cell-extrinsic factors (Additional file 1: Fig. S3i).

Intra-tumoral TCR clonality was reported to be associated with ICB response in recent studies [59]. We therefore applied the TRUST [60] algorithm on the tumor RNAseq data to infer the tumor-infiltrating immune repertoires. Different from regular TCR/BCR-seq approaches that sequence the immune repertoires directly from the genomic DNA, TRUST extrapolates immune repertoires from RNA-seq data. Responders had higher levels of recombined immune repertoires (Additional file 1: Fig. S4a-b), potentially due to their higher level of adaptive immune infiltration. Consistent with previous studies, we detected slightly higher TCR clonality and significantly higher BCR clonality in the responders (Additional file 1: Fig. S4c), supporting the role of clonal expanded $\mathrm{T}$ and $\mathrm{B}$ cells in tumor elimination. Furthermore, we applied our previously published computational method TIDE [41] on the tumor RNA-seq data to estimate the level of immunosuppressive cell types that can cause $\mathrm{T}$ cell exclusion (Fig. 3h, i). Consistent with the IHC and immune marker gene expression, $\mathrm{T}$ cell exclusion score is higher in non-responders than responders (Fig. 3h), showing positive correlation with gene expression signatures from myeloid-derived suppressor cells (MDSC) and M2 macrophages (Fig. 3i). We assessed the clinical relevance of the response-associated gene expression signature in mouse by assessing the association between signature score and patient survival in published ICB treatment cohorts with Cox-PH regression. We found that the mouse responder signature significantly correlated with a lower survival hazard (reflected by a negative Cox-PH regression coefficient value and $z$ score) in the majority of ICB clinical studies (Fig. 3j-1). Together, these results show that cancer 
cells originating from the same ancestors can form tumors of ICB responders or nonresponders.

\section{ICB treatment enriched ICB-resistant cancer clones in responders}

The assays above addressed the heterogeneity of response to ICB treatment at the bulk tumor level. We then focused on the response at the cancer clone level by isolating single cell-derived clones to investigate their phenotype (Fig. 4a).

We established $\sim 150$ single cell-derived lines from engrafted tumors by limiting dilution. Upon genotyping of the barcode in each line, we identified 5 lines (numbered A01, A06, B04, B64, and C32) representing 2 distinct barcoded clones that were significantly enriched in the ICB responders compared to the non-responders (Additional file 2: Table S1, Fig. 4b, Additional file 1: Fig. S5). These clones were also enriched in both anti-PD-1- and anti-CTLA-4-treated tumors compared to the IgGtreated control (Fig. 4c), suggesting they are more resistant to ICB treatment than the average of other clones. To verify this, we conducted another round of growth experiments on the B04 and B64 lines in vivo (each represented by different barcodes) and compared these to the parental line. The "parental line" used here and later experiments refers to the in vivo-derived bulk CT26 cells to control for engraftmentmediated selection and facilitate data comparison. Lines B04 and B64 manifested increased resistance to combined anti-PD-1/anti-CTLA-4 (Fig. 4d), demonstrating that the cancer clones enriched in the ICB responders were more resistant to ICB treatment. These results suggest that the ICB responders had a higher composition of ICBresistant cancer clones following ICB treatment. Together with the microenvironment characterization in Fig. 3, our data support a model that the responders had higher cancer cell-extrinsic antitumor immunity, and such selective pressure consequently drove clonal selection and enrichment of cancer cell-intrinsic ICB-resistant clones. In contrast, the non-responders lacked effective $\mathrm{T}$ cell infiltration and showed a lower level of clonal selection.

\section{ICB-resistant clones show distinct transcription signatures associated with $\mathrm{T}$ cell dysfunction}

We tried to understand the mechanisms of ICB resistance in clones B04 and B64 from different aspects. First, loss of antigen presentation or sensitivity to IFN $\gamma$ induction has been reported to confer resistance to immunotherapy [12, 16, 61]. We therefore compared the MHC-I and PD-L1 levels of B04, B64, and the parental line, but observed similar baseline levels and similar induction by IFN $\gamma$ (Additional file 1: Fig. S6a-b). This indicates that the resistance mechanisms of B04 and B64 are not due to lack of MHC-I expression or insensitivity to IFN $\gamma$.

Next, we investigated whether B04 and B64 lines are associated with T cell dysfunction. To this end, we borrowed insights from our previous TIDE study [41], which utilized a Cox proportional hazard (Cox-PH) model to identify gene expression signatures associated with $\mathrm{T}$ cell dysfunction in TCGA and PRECOG datasets. This signature reflects the gene expression in both the dysfunctional $\mathrm{T}$ cells and in other cells in the tumor which are associated with $\mathrm{T}$ cell dysfunction. We conducted RNA-seq analyses on B04, B64, and the parental line and observed a significant positive correlation 


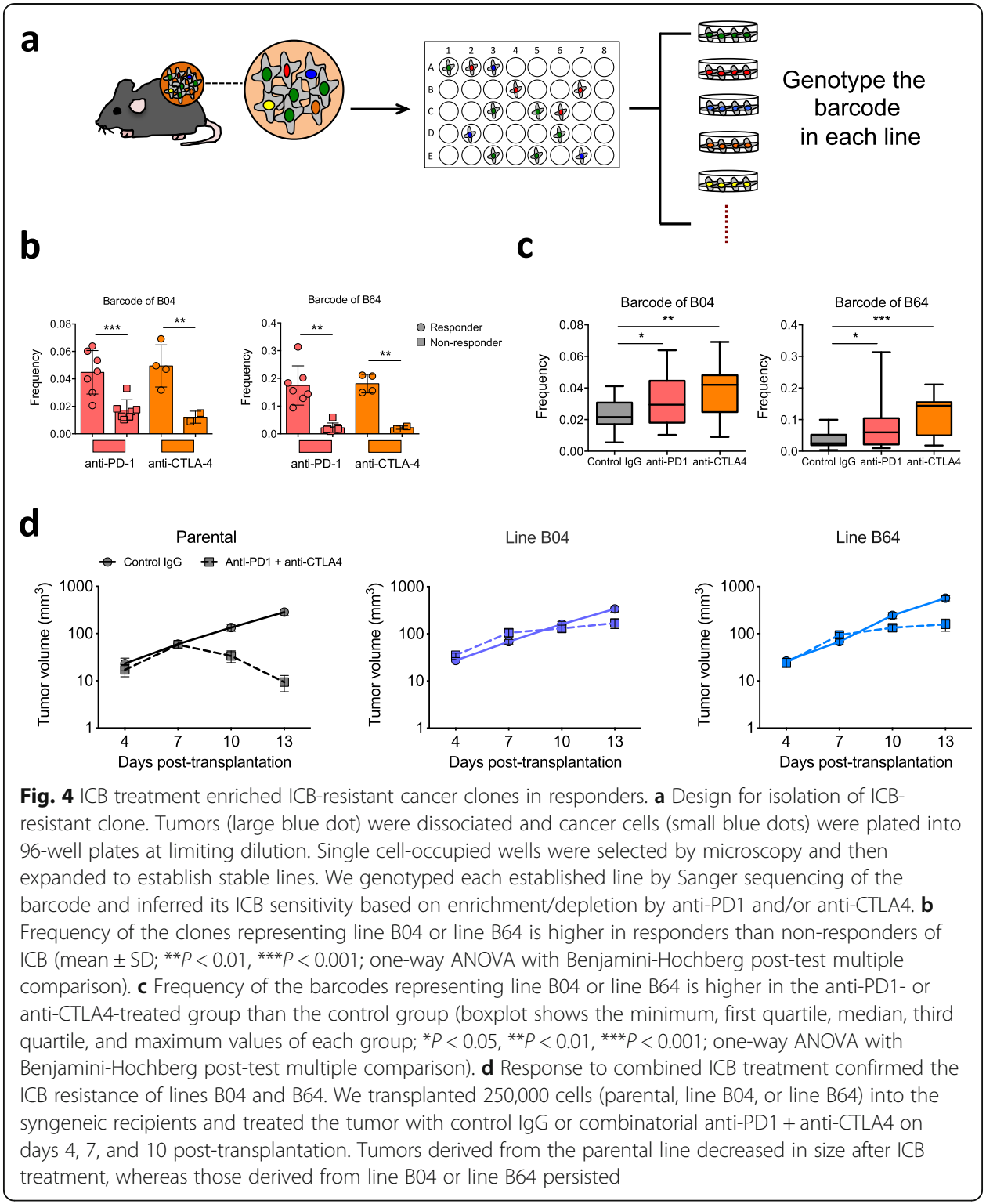

between differentially expressed genes in the resistant lines and the TIDE T cell dysfunction signature (Additional file 1: Fig. S7a). Genes with a strong positive TIDE dysfunction $z$ score, whose higher expression indicates cytotoxic $\mathrm{T}$ cell dysfunction, were upregulated in the ICB-resistant lines (Additional file 1: Fig. S7b), whereas those with negative TIDE $z$ score were unchanged or downregulated (Additional file 1: Fig. S7c). While this observation suggested that cancer cells with B04 and B64 signature might be associated with $\mathrm{T}$ cell dysfunction in clinical tumors, we wondered whether they might represent distinct mechanisms. Indeed, PCA of the RNA-seq profiles revealed that these two resistant lines have distinct gene expression changes compared to the parental line (Fig. 5a). Closer examination revealed that line B04 had higher expression of genes in DNA replication and sterol biosynthesis and lower expression of genes in ribosome biogenesis, whereas line B64 had higher expression of genes in interferon response and lower expression of genes in growth factor binding (Fig. 5b). We performed 


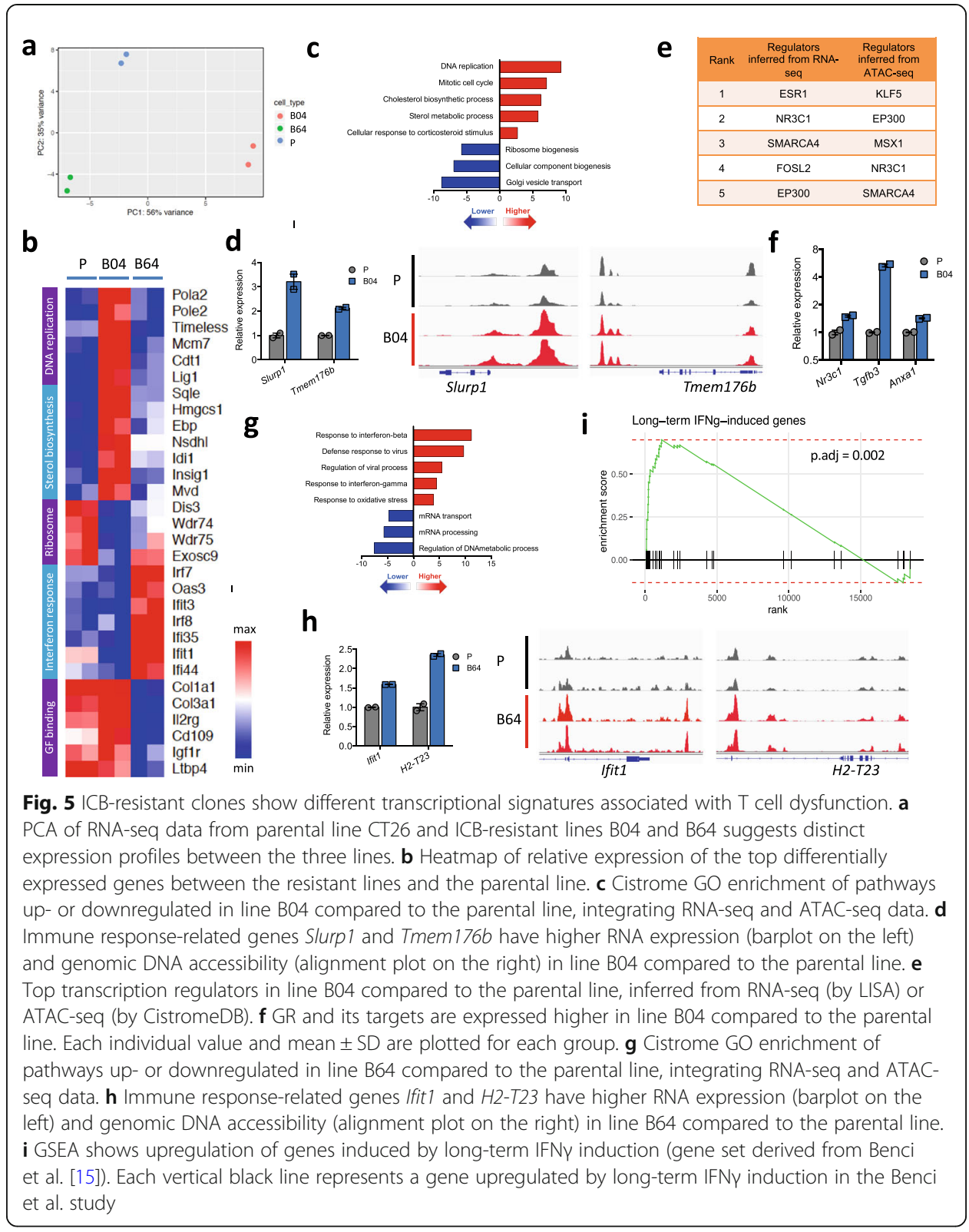

VarScan analysis of RNA-seq data from parental CT26 line, line B04, and line B64 to identify the genetic differences. We found a mutation in Arhgap30 (Rho GTPase Activating Protein, L458S) that was present in B04 but absent in parental line and B64. We did not find any B64-specific mutations. Arhgap30 was expressed in B04 but not expressed at detectable levels in parental line or B64, which likely explains why this SNP was detected only in B04. Although this gene is reported to be correlated with LDL cholesterol levels, we do not know what underlies the differential expression of this gene. We therefore speculate that the transcriptional differences in B04 and B64 might be driven by epigenetic factors.

To further characterize the differentially expressed genes in the resistant lines, we performed ATAC-seq to assess their differences in chromatin accessibility. Similar to RNAseq, PCA on the ATAC-seq data also suggests distinct epigenetic changes between line 
B04 and line B64 as compared to the parental line (Additional file 1: Fig. S7d). Using regulatory potential derived from our previously developed BETA algorithm [62] to assign the differential ATAC-seq peaks to genes, we found good correlation between differential ATAC-seq and differential RNA-seq (Additional file 1: Fig. S7e-f). We then used Cistrome GO [63] to find the most differentially regulated genes by combining the differential ATAC-seq and differential RNA-seq data. The upregulated genes in line B04 were enriched for cell cycle and sterol metabolic pathways from GSEA [63, 64] analysis (Fig. 5c) and included many genes known to be related to immune response. For example, Slurp1 and Tmem176b were upregulated in line B04 (Fig. 5d). SLURP1 is a secreted protein with immunomodulatory effects [65], and TMEM176B is a cation channel protein only recently discovered to suppress antitumor immunity via the inflammasome pathway [66]. To infer the putative transcription factors regulating the observed differential ATAC-seq and differential RNA-seq, we used two computational methods to exploit published ChIP-seq and chromatin accessibility profiles in Cistrome Data Browser (CistromeDB) [67]. For differential ATAC-seq, we used the toolkit function in CistromeDB [68] to identify transcription regulators whose published binding sites have the highest overlap with our differential ATAC-seq peaks. For differential RNA-seq, we used LISA [69] to first construct a chromatin model based on the differential genes and then identify the transcription regulators whose binding has the biggest effect on these genes based on the chromatin model. The two approaches retrieved consistent predictions, with nuclear receptor NR3C1, SWI/SNF complex component SMARCA4, and histone acetyltransferase EP300 as top candidates (Fig. 5e, Additional file 4: Table S3, Additional file 5: Table S4). In support of the $\mathrm{NR} 3 \mathrm{C} 1$ prediction, the expression of $\mathrm{Nr} 3 \mathrm{c} 1$ (encoding glucocorticoid receptor, GR) as well as its downstream targets was significantly upregulated in B04 (Fig. 5f). GR is known to mediate the immunosuppressive effects of glucocorticoids, and its agonists have been used clinically to suppress inflammation and relieve autoimmune side effects [70-72]. GR ChIP-seq from CistromeDB also revealed that GR can bind to the promoter and/or enhancer regions of Slurp1 and Tmem176b (Additional file 1: Fig. S7g) [73]. Activation of GR in cancer cells was reported to induce resistance to chemotherapy or targeted therapies [74-76], and our results suggest that GR might also induce resistance to immunotherapy.

Different from our observations in line B04, the most upregulated genes from combined differential RNA-seq/ATAC-seq data in line B64 were enriched for genes involved in response to type I and type II interferons (Fig. 5g), including genes Ifit1 and H2-T23 (Fig. 5h). IFIT1 is an interferon-stimulated gene with reported antiviral effects [77], and H2-T23 is a class Ib MHC that interacts with the NKG2A inhibitory receptor on NK cells and $\mathrm{T}$ cells to suppress their activation $[78,79]$. In addition, key transcription factors downstream of interferon, such as Irf7, Irf8, and Stat2, were upregulated in B64 and can bind to the promoter/enhancer region of Ifit1 and H2-T23 (Additional file 1: Fig. S7h-j). Recently, multiple studies reported that type I and II interferon signaling can compromise cancer cell response to $\mathrm{T}$ cell-mediated killing $[15,80]$, via mechanisms such as upregulation of $\mathrm{T}$ cell inhibitory receptor ligands (e.g., PD-L1 and MHC-II) and other cancerassociated inhibitory interferon-stimulated genes (e.g., IFIT1 and MX1). Indeed, the genes induced by long-term IFN $\gamma$ treatment in their study which were reported to be associated with ICB resistance were also enriched in the upregulated genes in line B64, including Ifit1 (Fig. 5h, i). 
In summary, these results suggest that different cancer clones, even those derived from the same parental line, can have distinct transcriptomic and epigenetic characteristics, and heterogeneous sensitivity to ICB treatment. These clones are subject to different selection pressures in different hosts, which likely underlie the diverse response patterns observed.

\section{Mathematical modeling of the clonal dynamics predicts that ICB-resistant clones are enriched in responders}

Our results above demonstrated that there are heterogeneous responses to anti-PD-1 and anti-CTLA-4 not only at the bulk tumor level but also at the clonal level. To quantitatively assess the contribution of clonal level ICB resistance to bulk tumor growth, we performed mathematical modeling of the barcoded clones in the tumors. We first established the theoretical framework of clonal selection in vivo by deducing the growth advantage of a clone compared to the bulk population [81]. This model assumed that the fitness advantage of each clone is reflected by its log-transformed frequency (Fig. 6a). We applied a Bayesian statistical inference method [83] to model clone selection under all treatment conditions and identified 6 clusters of clones with different growth patterns and sensitivity to ICB treatment (Fig. 6b, Additional file 1: Fig. S8a-c). Among them, there are clones more sensitive to both anti-PD-1 and antiCTLA-4 (clusters \#1, \#2, and \#3), and those more resistant to both anti-PD-1 and antiCTLA-4 (clusters \#5 and \#6). Consistent with experimental validation of resistance, lines B04 and B64 were assigned to the resistant clusters \#5 and \#6, respectively.

To examine the contribution of ICB-resistant clones to the ICB response in the bulk tumors, we compared the cumulative frequency of clones in each cluster among different tumors. Consistent with the observation for lines B04 and B64, resistant clones (those belonging to clusters \#5 and \#6) showed higher frequency in ICB responders than non-responders (Fig. 6c). Conversely, sensitive clones (those belonging to clusters $\# 1$, \#2, and \#3) showed higher frequency in non-responders than responders (Fig. 6c). When expanding the analysis to all tumors, we observed a negative correlation between ICB-resistant clone abundance and tumor size after ICB treatment (Additional file 1: Fig. S8d), and conversely a positive correlation between ICB-sensitive clone abundance and tumor size (Additional file 1: Fig. S8e). These results revealed that the nonresponders may actually contain a higher percentage of ICB-sensitive cancer cells after ICB treatment. It also implies that tumors with cancer cell-intrinsic resistance signatures in the on-treatment samples may correlate with better tumor response to ICB, suggesting that the tumors harbor sufficient immunity to eliminate the ICB-sensitive cancer cells. Interestingly, tumors treated by control IgG showed the same trend as those treated by ICB (Additional file 1: Fig. S8d-e), indicating that pre-existing intratumoral immune pressure might impose clonal selection of cancer cells even in the absence of ICB treatment.

To validate the clinical relevance of these observations from the mouse tumor model, we examined the bulk tumor RNA-seq data from multiple clinical studies [11, 20, 35, 54, 82, 84]. In the two studies where both pre-treatment and on-treatment tumor RNA-seq data are available for comparison [54, 82], the B04 and B64 gene expression signatures are significantly associated with better ICB response in the on/post-treatment tumors (Fig. 6d, e), but 


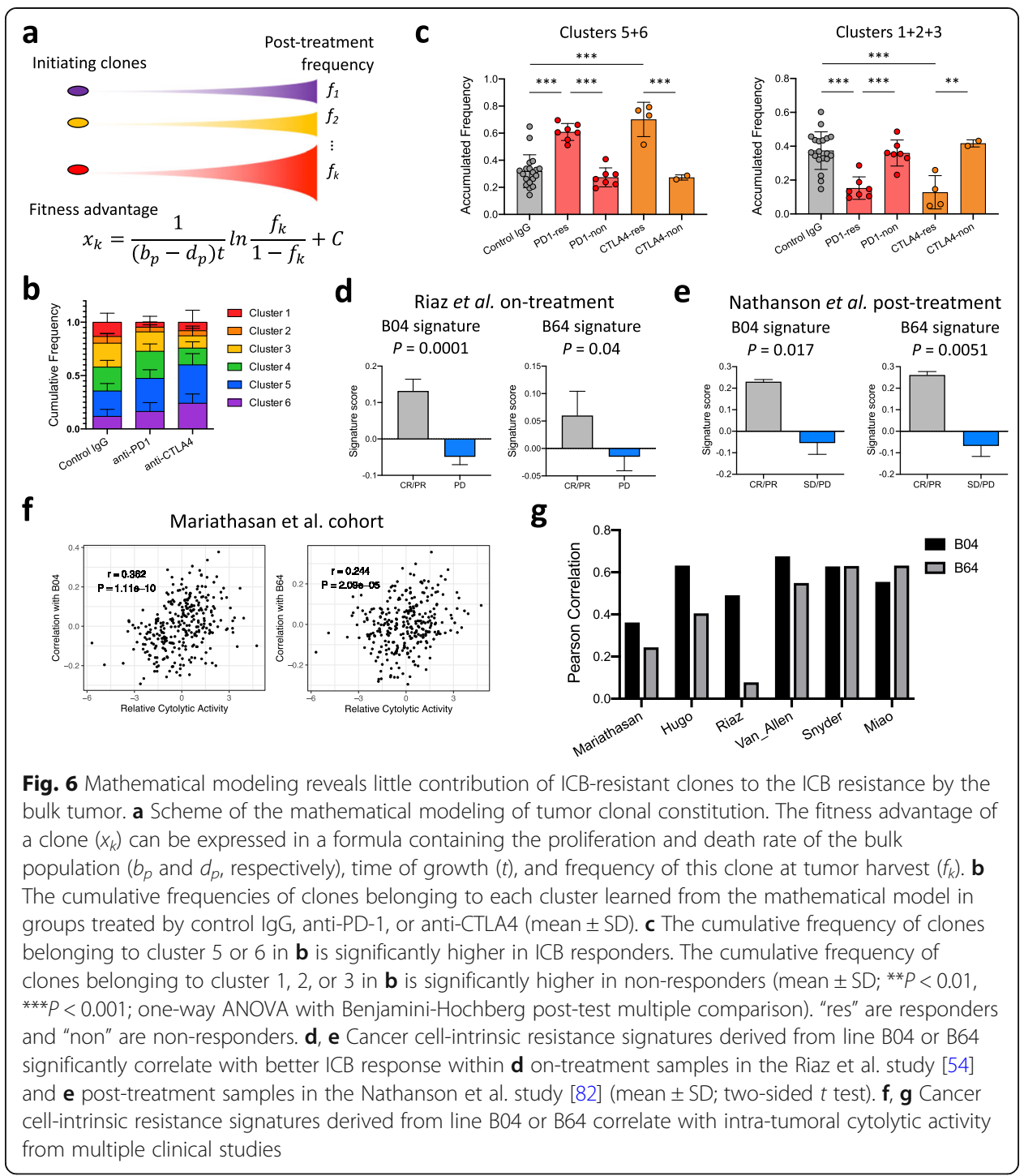

not the pre-treatment ones (Additional file 1: Fig. S8f-g). This is consistent with our observation in the mouse that the resistant clones were enriched after response to ICB treatment. Furthermore, in other studies where only pre-treatment RNA-seq data is available, B04 and B64 signatures show positive correlation with microenvironmental cytolytic activity based on transcription of GZMA and PRF1 [85] (Fig. 6f, g). This observation supports our computational modeling that the presence of cancer cell-intrinsic immune resistance signature in tumors prior to immunotherapy treatment is an indication of pre-existing immune pressure and cytolytic activities.

Taken together, our approach combining a unique mouse system and extensive clinical data mining from multiple cohorts demonstrates that tumors originating from the same group of cancer cells can show diverse response patterns to ICB. Therefore, in addition to mutation profiling at diagnosis, characterizing cancer clonal constitution and the tumor microenvironment during treatment may provide complementary insights to improve patient outcomes. 


\section{Discussion}

Heterogeneous response to ICB among different patients remains a major challenge of cancer immunotherapy. Numerous clinical studies have been performed to assess treatment efficacy and response biomarkers $[1-3,10,11,20,53,54]$. However, it is difficult to identify consistent resistance patterns, likely due to the remarkable inter- and intratumor heterogeneity. In this study, we identified heterogeneous responses to ICB treatment in multiple syngeneic transplantable tumor models and further identified diverse response patterns of the same cancer clones in tumors with different responses to ICB.

Comparing tumor growth and barcode distribution across different mouse tumors within the same treatment group, we demonstrated that response can differ dramatically between tumors with the same initial clonal constitution. Good response to ICB is strongly associated with elevated microenvironmental immune infiltration and $\mathrm{T}$ cell activation. Such microenvironment-dominated signature is associated with good prognosis in all but one clinical cohort tested. Comparing barcode frequencies between different treatment groups followed by clonal validation, we found that ICB-resistant cancer clones exist in the bulk cancer cell population prior to ICB treatment. We isolated two ICB-resistant cancer clones for further study and discovered that they have different transcriptional and epigenetic profiles and harbor distinct ICB-resistance mechanisms. Line B04 has elevated expression of genes downstream of nuclear receptors, such as GR. Glucocorticoid signaling was reported to mediate diverse effects in both cancer cells and immune cells. GR agonists, such as dexamethasone, can inhibit the activation and proliferation of lymphocytes, thus suppressing inflammation [70,71, 86]. On the other hand, elevated GR signaling in cancer cells can mediate resistance to targeted therapy and promote cancer metastasis [74-76, 87]. Further investigation of GR is needed to uncover its role in immunotherapy response. Line B64 has elevated basal level of interferon response signature. This was surprising because interferon signaling is critical for $\mathrm{T}$ cell-driven inflammation and response to ICB treatment $[12,16]$, so increased interferon response signature was not expected to be associated with ICB resistance. However, recent studies also revealed that prolonged interferon exposure can regulate a multigenic resistance program to ICB [15], and indeed, we found genes involved in such resistance program to be upregulated in B64. In addition, elevated interferon signaling has also been reported to mediate resistance to chemotherapy, radiotherapy, or targeted therapies [88-90].

Built upon barcode distributions, our mathematical modeling revealed that ICB responders, but not non-responders, may show enrichment of cell-intrinsic ICBresistance signatures during ICB therapy. Our simulation result comports with analyses of clinical data that ICB responders, rather than non-responders, show an enrichment of cancer cell-intrinsic resistance signature post-treatment. Consequently, the presence of ICB-resistant cancer clones is more likely to contribute to the late-developing resistance in the initial ICB responders, rather than the primary resistance in the initial nonresponders. This finding marks a critical difference between immunotherapy and targeted therapies and emphasizes the critical role of cancer cell-extrinsic factors in ICB response. Systematic examination of the abundance of ICB-resistant clones and characterization of tumor microenvironment in ICB responders or non-responders corroborates the mathematical simulation and confirms the critical role of tumor microenvironment in ICB response. Our result indicates that genetic biomarkers alone may not be able to completely distinguish ICB responders from non-responders. Instead, an 
expression signature that reflects both the cancer cells and the immune microenvironment might provide important insights to ICB response prediction.

Despite the intriguing observations from our study, it remains elusive how different tumor microenvironments arise between different recipients of the same cancer cell line. Previous studies revealed multiple cancer cell-intrinsic mechanisms that can modulate the tumor microenvironment [14, 21, 57, 91-94]. However, in our study, we transplanted the same cancer cell line, so the difference in microenvironment between ICB responders and non-responders is unlikely attributable to cancer cell-intrinsic factors. Other studies suggested that the gut microbiota in each individual may play a critical role in their immune system development and ICB response [37, 95, 96]. In our study, all mice were obtained from the same company and maintained in the same facility, and we did not observe significant differences between cages, so the difference in ICB response we observed might not be attributable to the gut microbiota. One possible explanation is the general immune health of each individual at the time of tumor establishment and ICB treatment, a comprehensive state influenced by diet, exercise, immune repertoire, mental health, and other factors which merit further study. Another limitation of our study is that we isolated the single cell-derived cancer clones after, rather than before, ICB treatment. This is because all cancer clones had very low initial frequencies and ICB treatment provided a means to enrich ICB-resistant clones for isolation. Consequently, the transcriptome and epigenetic changes in lines B04 and B64 might be either pre-existing prior to ICB treatment or induced by ICB treatment. In either scenario, our data indicate that there are several pathways by which cancer cells may become ICB-resistant. Furthermore, as is the limitation with most cancer models in mice, the speed of disease development in transplantation models usually precludes the feasibility of assessing the role of mutation accumulation in the process of tumor development and ICB response. We expect that genomic engineering tools can be integrated with these models to simulate mutation accumulation and clonal evolution along tumor development and treatment response.

Whereas both cancer cell-intrinsic factors and cancer cell-extrinsic factors are known to influence the response to immunotherapy, our study found that even for the same labeled cancer cells grown in isogenic individuals, the immune response can be diverse. This finding has two important implications for clinical treatment and translational research. First, our results indicate that there is an inherent limitation in using pre-treatment genetic information to guide immunotherapy treatment decisions because tumors with a defined genetic background may show heterogeneous responses. Therefore, longitudinal data on the tumor microenvironment, such as by RNA-seq of biopsies, or monitoring of patient general immune health, such as by examining peripheral blood, will likely help optimize treatment strategies. Second, our study reveals a paradoxical observation, which may affect interpretations of tumor RNA-seq data from studies associating ICB response with expression signatures. When studying biomarkers for ICB response or resistance, it is common to use RNA-seq from tumors that respond to ICB as the "responder" signature. Our study suggests that immune-resistant cancer cells are enriched in such samples. Therefore, the "responder" signature derived this way may in fact be enriched for a cancer cell-intrinsic resistance signature, the opposite of the 
intended result. This phenomenon may thereby contribute to the variable performance of ICB response biomarkers in different clinical cohorts.

\section{Conclusions}

In this study, we sought to address the heterogeneity of response to ICB. From analysis of published ICB clinical trials, we found that each biomarker shows variable prediction power in different studies. To further assess the source of such heterogeneity, we performed clonal tracing and mathematical modeling of cancer clonal dynamics in response to ICB. We found that the same population of cancer clones can show diverse response patterns in different tumors. ICB responders showed higher immune infiltration and enrichment of ICB-resistant clones post-treatment compared to nonresponders. The cancer cell-intrinsic ICB-resistance signatures in on-treatment/posttreatment samples are correlated with better ICB response. These results demonstrate that tumors originating from the same cancer cells can show heterogeneous responses to ICB depending on the tumor microenvironment; therefore, longitudinal data on the tumor microenvironment or patient immune health may complement mutation profiling to optimize treatment. Furthermore, because ICB responders may be enriched for cancer cell-intrinsic resistant signatures, our study suggests that deconvolution of tumor RNA-seq data into cancer cell-intrinsic/extrinsic signatures may be required for proper interpretation of response-signature association studies.

\section{Methods}

Mice

All mice were housed in standard cages in Dana-Farber Cancer Institute Animal Resources Facility (ARF). All animal procedures were carried out under the ARF Institutional Animal Care and Use Committee (IACUC) protocol and were in accordance with the IACUC standards for the welfare of animals. Wild-type Balb/c and C57BL/6 recipient mice were purchased from Charles River laboratory.

\section{Barcoding of cancer cells}

CT26 mouse colorectal cancer cell line was cultured in RPMI-1640 medium supplemented with $10 \%$ FBS and 1\% penicillin-streptomycin. We transduced the CT26 cells with the ClonTracer barcode retroviral library using $8 \mu \mathrm{g} / \mathrm{ml}$ polybrene. After 24-h incubation, infected cells were selected with puromycin. To ensure that the majority of cells were transduced with a single virion, we performed the transduction at low multiplicity of infection $(\mathrm{MOI}<0.05)$. To avoid marking multiple clones with the same barcode, we restricted the number of cells infected during the transduction $(<10,000)$ prior to the follow-up expansion (Supplementary Fig. 1a). Expanded cultures from the same batch of infection were highly consistent in barcode distribution (Supplementary Fig. 1b), enabling systematic assessment of enrichment or depletion of clones in response to drug treatment.

\section{Barcode amplification and sequencing}

At the end of ICB treatment in vivo, we harvested residual tumors from each recipient mouse and extracted bulk genomic DNA with the AllPrep DNA/RNA Mini Kit 
(Qiagen) for barcode amplification. We used PCR to amplify the barcode sequence for NGS with protocols as previously described [51]. We sequenced the PCR-amplified barcode library on the Illumina HiSeq-2500 sequencer, with paired-end 150 TruSeq kit. Deconvolution of sequencing results and quantification of barcode composition were performed by clonTracer_v1.2 software [51].

\section{Assess the heterogeneity of ICB response between different tumors and different cancer} clones

We transplanted $2.5 \times 10^{5}$ barcoded CT26 cells subcutaneously into the left and right flank of 6-8-week-old female Balb/c mice (from Charles River Laboratory). When the tumors became palpable (day 7 post-transplantation), we began treatment with control IgG (clone 2A3), anti-PD1 (clone 1A12), or anti-CTLA4 (clone 9D9), by intraperitoneal injection $(200 \mu \mathrm{g}$ per mouse in $200 \mu \mathrm{l} \mathrm{HBSS}$ buffer) every 3 days for a total of 5 times. Three days after the last treatment, we harvested tumors for immunohistochemistry and DNA/RNA extraction.

To assess the heterogeneity of ICB response between different tumors, we monitored tumor size along the course of treatment and identified "responder" or "non-responder" tumors in the anti-PD1 or anti-CTLA4 cohorts based on their size at the end of treatment compared to the tumors in the control IgG cohort. Specifically, tumors larger than $10^{M-0.1}$ were classified as "non-responder" and tumors smaller than $10^{M-0.5}$ were classified as "responder" ( $M$ denotes the logarithmic median tumor size in the control IgG cohort). We also applied an alternative approach to classify responder and nonresponder by the magnitude of change between post- and pre-treatment tumor size, which led to similar results. We then performed immunohistochemistry and bulk tumor RNA-seq to characterize the difference between responders and non-responders. Notably, there were 8 tumors in the anti-CTLA4 cohort with complete response, which prevented us from profiling their tumor microenvironment.

To evaluate the intra-tumoral heterogeneity of cancer clones in response to ICB treatment, we used the genomic DNA harvested from each tumor at the end of treatment course for barcode amplification and sequencing. We compared global barcode composition in all samples by hierarchical clustering. For each barcode, we compared its logtransformed frequency in control IgG, anti-PD1, or anti-CTLA4 cohort by ANOVA.

\section{Assess the heterogeneity of ICB response in other syngeneic models}

A total of 250,000 MC38, 4T1, or EMT6 cells were transplanted subcutaneously (for MC38) or into the mammary fat pad (for 4T1 and EMT6) into syngeneic recipients. When the tumors grew to $100-200 \mathrm{~mm}^{3}$ in size, we started control IgG or anti-PD1 treatment $100 \mu \mathrm{g}$ per mouse every 3 days for a total of 4 doses. We monitored tumor size along the treatment course and compared the intra-group variance between the control IgG and anti-PD1 groups in each model by the $F$ test of the equality of variances.

\section{Isolation of ICB-resistant single cell-derived clones}

We first transplanted $2.5 \times 10^{5}$ barcoded CT26 cells into syngeneic Balb/c recipient mice for tumor establishment in vivo. On day 16 post-transplantation, after the tumor 
was established in vivo, we harvested the bulk tumor for isolation of single cell-derived clones and then genotyped each clone for their barcode identity. Specifically, tumors were cut into small pieces, digested in collagenase/hyaluronidase for $20 \mathrm{~min}$ at $37^{\circ} \mathrm{C}$, washed with RPMI media, and filtered through a $70-\mu \mathrm{m}$ cell strainer. Cells were resuspended in RPMI medium supplemented with $10 \%$ FBS and 1\% penicillin/streptomycin for culture. The next day, we started treatment of the cell culture with $10 \mu \mathrm{g} / \mathrm{ml}$ puromycin for 2 days to select barcoded CT26 cells.

To acquire single cell-derived clones, we plated the barcoded CT26 cells from the previous step into 96-well plates at limiting dilution and selected wells occupied by a single cell under the microscope for further culture. We then extracted genomic DNA from each single cell-derived clone and sequenced its barcode for identification. Each single cell-derived clone was evaluated for ICB sensitivity/resistance based on the depletion/enrichment of its corresponding barcode by anti-PD1 or anti-CTLA4 treatment.

\section{Validation of ICB resistance in isolated lines}

As above, we cultured bulk barcoded CT26 cells isolated from established tumors as control "parental" line and expanded the resistant lines \#B04 and \#B64, which harbored the barcodes enriched by both anti-PD1 and anti-CTLA4 treatment. To verify the ICB resistance of line B04 and line B64, we transplanted $2.5 \times 10^{5}$ parental, line B04, or line B64 cells into 6-8-week-old Balb/c recipient mice and performed combination ICB or single agent treatment for validation. For single agent treatment, we used $200 \mu \mathrm{g}$ control IgG (clone 2A3), anti-PD1 (clone 1A12), or anti-CTLA4 (clone 9D9) per mouse each time. For anti-PD1/anti-CTLA4 combination treatment, we used $200 \mu \mathrm{g}$ control IgG per mouse in the control treatment group and $100 \mu \mathrm{g}$ anti-PD1 $+100 \mu \mathrm{g}$ antiCTLA4 in the ICB treatment group per mouse each time.

All mouse studies were approved by IACUC at Dana-Farber Cancer Institute and performed in accordance with the IACUC standards for the welfare of animals.

\section{Flow cytometry}

For assessment of MHC-I and PD-L1 levels, CT26 cells (in vivo-selected parental control or isolated ICB-resistant lines) were treated by vehicle control or $10 \mathrm{ng} / \mathrm{ml} \mathrm{IFN \gamma}$ for $48 \mathrm{~h}$. Cells were then dissociated and resuspended in PBS-2\%FBS and incubated with DAPI, anti-PD-L1 (clone MIH5, BD Biosciences), and anti-H2- $\mathrm{K}^{\mathrm{d}}$ (clone SF1-1.1, BioLegend) for $1 \mathrm{~h}$ on ice. Cells were then washed, resuspended in PBS-2\%FBS, and analyzed on BD LSR-Fortessa instrument. FACS data was then analyzed by FlowJo software.

For assessment of intra-tumoral immune infiltration in the MC38 model, 9-week-old C57BL/6 (Charles River) mice were implanted with $1 \times 10^{5}$ MC38 cells in each flank. On days 14, 16, and 18 post-tumor implantation, animals received intraperitoneal injection of anti-PD-1 monoclonal antibody (clone 29F.1A12, $50 \mu \mathrm{g} / \mathrm{animal}$ ). Animals were euthanized on day 32 post-tumor implantation, and the resulting tumors were dissected, digested (Miltenyi mouse tumor dissociation kit, 130-096-730), depleted of red blood cells by ACK solution lysis, and stained with fluorescent conjugated antibodies for flow cytometry analysis for tumor-infiltrating immune cell populations using an 
established antibody panel and flow cytometry protocol [97]. We used the summed frequency of $\mathrm{CD} 4+$ and $\mathrm{CD} 8+\mathrm{T}$ cells in all viable singlet cells to quantify $\mathrm{T}$ cell infiltration in each sample.

\section{Immunohistochemistry}

Immunohistochemistry of FFPE samples of mouse tumors was performed at the Brigham and Women's Hospital (BWH) Specialized Histopathology Core Lab.

\section{RNA-seq}

For comparison of transcription profile between single cell-derived clones and the parental line, we extracted total RNA from in vivo-selected parental line or isolated ICBresistant lines (\#B04 and \#B64) cultured in complete RPMI-1640 culture medium in duplicates. RNA extraction was performed using the miRNeasy Plus Mini Kit (Qiagen, \#17004) following the manufacturer's protocol. Total RNA was submitted to Novogene Inc. for sequencing. Standard mRNA library preparation kit was used for library preparation. Paired-end $150 \mathrm{bp}$ sequencing was done on Illumina HiSeq 2500. RNA-seq fastq reads were aligned to the mm10 genome using STAR v2.5 [98] with default settings and quantified using RSEM [99] with default settings. The downstream differential gene expression analysis was performed using DESeq2 [100], with default settings, and differentially regulated gene sets were derived by GSEA [64] with default settings. To identify genetic mutations in the barcoded cell lines, we performed VarScan [101] analysis of parental CT26 line, line B04, and line B64 RNA-seq with default settings.

For comparison of transcription profile between tumor samples, we extracted total RNA from bulk tumor samples using the AllPrep DNA/RNA Mini Kit (Qiagen, \#80204). Total RNA was submitted to Novogene Inc. for sequencing as above.

\section{ATAC-seq}

For comparison of chromatin accessibility between single cell-derived clones and the parental line, ATAC-seq was performed using in vivo-selected parental line or isolated ICB-resistant lines (\#B04 and \#B64) in duplicates (200,000 cells) by the Center for Functional Cancer Epigenetics at DFCI as previously described. For data analysis, we used Burrows-Wheeler Aligner (BWA) to map sequencing reads to the reference genome and MACS2 for peak calling. DESeq2 was applied to identify the differentially accessible regions between ICB-resistant lines and the parental control line.

\section{Infer transcriptional regulators from RNA-seq}

We inferred the transcriptional regulators underlying the transcriptional difference between the parental line and ICB-resistant lines by the LISA algorithm (http://lisa.cistrome.org/). Briefly, given a differentially expressed gene set, LISA uses DNase-seq and H3K27ac ChIP-seq data in public databases to model the chromatin landscape of the cell type and lineage context. Based on the chromatin landscape, LISA uses two methods to infer the relevant transcription regulators by evaluating all the ChIP-seqdetermined transcription regulator binding sites.

With direct ChIP method, for each transcription regulator ChIP-seq dataset, LISA examines the regulatory potential for the target gene set compared to background gene 
set. The transcription regulators with largest difference in regulatory potential between target and background gene sets are reported as the driving transcription regulators.

With in silico deletion method, LISA first selects a small number of DNase-seq or H3K27ac ChIP-seq samples from the CistromeDB based on chromatin regulatory potential to discriminate the target gene set from a random background gene set. It then evaluates each putative transcription regulator cistrome by first setting the DNase-seq/ H3K27ac ChIP-seq chromatin signal to zero on the cistrome peaks and then testing whether it influences regulatory potential for target and background gene set. The transcription regulators that influence target gene set more than background gene set are reported as the driving transcription regulators.

The above two methods generated very similar results for our differentially expressed gene sets. We report the results for in silico deletion method in this manuscript.

\section{Infer transcriptional regulators from ATAC-seq}

We inferred transcriptional regulators associated with the differential ATAC-seq peaks by testing the significance of overlap with publicly available ChIP-seq peaks for each candidate transcription regulator (http://dbtoolkit.cistrome.org/).

\section{Integrative analysis of RNA-seq and ATAC-seq}

We integrated the results of RNA-seq and ATAC-seq to identify significantly regulated biological processes by Cistrome GO (http://go.cistrome.org/). Briefly, Cistrome GO first ranks genes by magnitude of change in transcription (using RNA-seq results) or change in regulatory potential (using ATAC-seq) and then integrates the two rankings for each gene into one ranking score. Finally, Cistrome GO performs GSEA analysis on integrated gene ranking for enriched GO terms.

\section{Gene set enrichment analysis}

GSEA was performed by software from Broad Institute, with gene sets from MSigDB as reference.

\section{Extrapolation of immune repertoire}

We extracted the CDR3 sequence and relative abundance of T/B cell receptors TRA/ TRB and IGH/IGL/IGK by TRUST [60, 102].

\section{TIDE T cell dysfunction/exclusion signature scores}

We calculated $\mathrm{T}$ cell dysfunction and exclusion scores by TIDE as previously described [41]. For $\mathrm{T}$ cell dysfunction scores, we used the Cox-PH model to test how the expression of a candidate gene interacts with the level of cytotoxic $\mathrm{T}$ cell infiltration in affecting death hazard. For T cell exclusion scores, we calculated the Spearman correlation of each sample's transcriptome with the reference transcriptome of MDSC, M2 macrophage, or cancer-associated fibroblast.

\section{Mathematical modeling of the cancer clonal dynamics in vivo}

We established a theoretical framework to simulate the selection of ICB-resistant clone, adapting a published model on subclonal selection in cancer [81]. We denote the 
proliferation rate and death rate of the bulk cancer cell population as $b_{p}$ and $d_{p}$, respectively. For a specific cancer clone $k$, we denote the proliferation and death rate as $b_{k}$ and $d_{k}$, respectively. The fitness advantage of clone $k\left(x_{k}\right)$ can be defined by the ratio of net growth rates between this clone and the background host population:

$$
1+x_{k}=\frac{b_{k}-d_{k}}{b_{p}-d_{p}}
$$

When $x>0$, the corresponding cancer clone has growth advantage over the average of the bulk population, under the condition tested.

We denote the frequency of the ICB-resistant clone as $f_{k}$ and the time of growth in vivo as $t$. The frequency of clone $k$ at time of tumor harvest can be deduced as:

$$
f_{k(\text { end })}=\frac{f_{k(\text { start })} \cdot e^{\left(b_{k}-d_{k}\right) t}}{f_{k(\text { start })} \cdot e^{\left(b_{k}-d_{k}\right) t}+\left(1-f_{k(\text { start })}\right) \cdot e^{\left(b_{p}-d_{p}\right) t}}
$$

Because several hundred clones successfully engrafted in each mouse, we can assume that $f_{k(\text { start })}<<1$. Combining Eqs. (1) and (2), we can deduce the value for relative growth advantage as:

$$
x_{k}=\frac{1}{\left(b_{p}-d_{p}\right) t}\left(\ln \frac{f_{k(\text { end })}}{1-f_{k(\text { end })}}+\ln \frac{1}{f_{k(\text { start })}}\right)
$$

For a specific cancer clone $k$, its frequency in the tumor at the start of treatment should be the same across different treatment groups. According to Eq. (3), the fitness advantage of this clone under each treatment condition should be proportional to $\ln$ $\frac{f_{k(\text { end })}}{1-f_{k(\text { end })}}$.

\section{Detection of different growth patterns}

We fitted the frequencies of barcoded cancer cells into a finite mixture of linear regression models to capture the detailed tumor heterogeneity. Our barcoding experiment ensured that each barcode represented a single clone and clones with the same genomic profile could be labeled by multiple barcodes. Assume that there are $K$ unique clonotypes in the tumor:

$$
h(y \mid x, \psi)=\sum_{k=1}^{K} \pi_{k} f\left(y \mid x, \theta_{k}\right), \text { s.t. } \pi_{k} \geq 0, \sum_{k=1}^{K} \pi_{k}=1
$$

where the cell growth rate $y$ depends on cell intrinsic and extrinsic factors $x$ with the density function $h()$, and $\psi$ includes the parameter estimations on the prior probability $\pi_{k}$ of component $k$ and the component-specific parameter $\theta_{k}$. Since we repeatedly measured the barcode changes with $N_{m}$ observations from barcode $m$, the log-likelihood of the mixture model becomes:

$$
\log L=\sum_{m=1}^{M} \sum_{n=1}^{N_{m}} \operatorname{logh}\left(y_{m n} \mid x_{m n}, \psi\right), \sum_{m=1}^{M} N_{m}=N
$$

We used the FlexMix package in $\mathrm{R}$ to optimize the log-likelihood and compute the posterior probability of barcode $m$ belonging to clonotype $k$. We define that the log of 
barcode frequency follows the Gaussian distribution with linear factors from the treatment condition and the log of tumor size.

\section{Evaluate the correlation of cancer cell-intrinsic or cancer cell-extrinsic signatures to clinical ICB response}

Similar to the TIDE $\mathrm{T}$ cell dysfunction/exclusion estimation, we calculated the line B04/B64 signature scores of bulk tumors as the Pearson correlation between bulk tumor gene expression and line B04/B64 gene expression. We first defined B04 (or B64) gene signature as the $\log _{2}$-fold-change of genes in each line compared to the parental line. For each tumor within one clinical cohort (e.g., the Riaz pre-treatment cohort), we normalized $\log _{2}$-TPM of gene $i$ in sample $j$ as the deviation from the mean $\log _{2}$-TPM of gene $i$ in all samples in the cohort:

$$
E_{i, j}^{\prime}=E_{i, j}-\bar{E}_{i}
$$

To derive the B04 (or B64) signature score in a tumor sample, we calculated the Pearson correlation between the reference signature (the $\log _{2}$-fold-change from the first step) and the normalized gene expression in the tumor sample $\left(E_{i, j}^{\prime}\right)$. If a sample has high expression of genes that are also highly expressed in line B04 (or B64), and low expression of genes that are also lowly expressed in line B04 (or B64), then this sample should have a high B04 (or B64) signature score. We used the 200 most up/downregulated genes in B04 and B64 for signature score calculation in order to focus on the genes that define the difference between these lines and the parental line and eliminate the noise that can be introduced by the genes with low expression variance. For pretreatment or on-treatment samples in each clinical cohort, we compared the Pearson correlation values between CR/PR patients and PD patients by Student's $t$ test. For pretreatment samples, we also compared the Pearson correlation coefficients and the cytolytic activity score of each patient and found a significantly positive correlation in the majority of studies. We performed robustness test (e.g., using the top 200, 400, 800, or all significantly differentially expressed genes between line B04/B64 and the parental line) and found the conclusion to be robust under different parameters.

Similarly, for cancer cell-extrinsic factors, we first calculated the differential gene expression between ICB responders and non-responders and included the top 200 most regulated genes to get the cancer cell-extrinsic signature. We computed the Pearson correlation between the cancer cell-extrinsic signature and the transcriptome of each patient in each clinical cohort. For each cohort, we calculated the association between patient survival and signature score (treated as a continuous variable) by Cox-PH regression.

\section{Supplementary information}

Supplementary information accompanies this paper at https://doi.org/10.1186/s13059-020-02166-1.

Additional file 1. Supplementary Figures.

Additional file 2: Table S1. Barcode identity of the isolated single-cell-derived clones.

Additional file 3: Table S2. Enriched pathways in responders vs non-responders by GSEA.

Additional file 4: Table S3. Cistrome Toolkit inference of transcription regulators with highest overlap with differential ATAC-seq peaks. 
Additional file 5: Table S4. LISA inference of transcription regulators that may best account for the differentially expressed genes.

Additional file 6. Review history.

\section{Acknowledgements}

We thank Kai Wucherpfennig, Zach Herbert, and Cristina Guarducci for their helpful discussions during manuscript preparation and revision.

\section{Review history}

The review history is available as Additional file 6.

\section{Peer review information}

Anahita Bishop was the primary editor of this article and managed its editorial process and peer review in collaboration with the rest of the editorial team.

\section{Authors' contributions}

SG conceived this project. SG and XW performed most of the experiments and analyzed the experimental results. XH performed the computational analysis. PJ and JF performed the analysis on T cell dysfunction and exclusion. ZL, NT, and XX helped with establishing single cell-derived clones. $Z \mathrm{~L}, \mathrm{XB}$, and $\mathrm{QT}$ helped with the in vivo ICB treatment studies. CW and ZZ helped with the analysis of historical scRNA-seq data. CM, JW, WZ, YZ, and BK provided critical help on scientific discussions. KL, PC, and HL performed the ATAC-seq experiment and analysis. XSL, MB, and GJF jointly supervised the study and wrote the manuscript together with SG. The authors read and approved the final manuscript.

\section{Funding}

The work was supported by grants from the NIH (R01 CA234018 and U01 CA226196 to XSL and P50 CA206963 and P50 (A101942 to GJF).

\section{Availability of data and materials}

All sequencing data were uploaded to Gene Expression Omnibus with accession number GSE139476 [103]. All analysis codes were uploaded to the GitHub repository: https://github.com/Shengqing-Stan-Gu/2020_Clone_Tracing_paper [104] and Zenodo: https://doi.org/10.5281/zenodo.4016933 [105]. The source code is licensed under the MIT license. The following public datasets were used: Mariathasan [35], Van Allen [11], Riaz [54], Snyder [55], Miao [84], Gide [53], Hugo [20], Nathanson [82], and Benci [15].

\section{Ethics approval and consent to participate}

All animal procedures were carried out under the DFCI Institutional Animal Care and Use Committee (IACUC) protocol and were in accordance with the IACUC standards for the welfare of animals.

\section{Consent for publication}

Not applicable.

\section{Competing interests}

XSL is a cofounder, board, and SAB member of GV20 Oncotherapy and its subsidiaries; SAB of 3DMed Care; consultant for Genentech; and stock holder of BMY, TMO, WBA, ABT, ABBV, and JNJ, and received research funding from Takeda and Sanofi.

$\mathrm{MB}$ is a consultant to and receives sponsored research support from Novartis. MB is a consultant to and serves on the scientific advisory boards of Kronos Bio, H3 Biomedicine, and GV20 Oncotherapy.

GJF has patents/pending royalties on the PD-1/PD-L1 pathway from Roche, Merck MSD, Bristol-Myers-Squibb, Merck KGA, Boehringer-Ingelheim, AstraZeneca, Dako, Leica, Mayo Clinic, and Novartis. GJF has served on advisory boards for Roche, Bristol-Myers-Squibb, Xios, Origimed, Triursus, iTeos, NextPoint, IgM, Jubilant, and GV20. GJF has equity in Nextpoint, Triursus, Xios, iTeos, IgM, and GV20.

\section{Author details}

'Department of Data Science, Dana-Farber Cancer Institute, Boston, MA 02215, USA. ${ }^{2}$ Department of Biostatistics, Harvard T.H. Chan School of Public Health, Boston, MA 02215, USA. ${ }^{3}$ Center for Functional Cancer Epigenetics, Dana-Farber Cancer Institute, Boston, MA 02215, USA. ${ }^{4}$ Department of Medical Oncology, Dana-Farber Cancer Institute, Boston, MA 02215, USA. ${ }^{5}$ Harvard Medical School, Boston, MA 02115, USA. ${ }^{6}$ Clinical Translational Research Center, Shanghai Pulmonary Hospital, School of Life Science and Technology, Tongji University, Shanghai 200433, China. ${ }^{7}$ Division of Gastrointestinal Cancer Translational Research Laboratory, Peking University Cancer Hospital \& Institute, Beijing 100142, China. ${ }^{8}$ University of Texas Southwestern Medical School, Dallas, TX 75390, USA.

Received: 7 April 2020 Accepted: 15 September 2020

Published online: 15 October 2020

\section{References}

1. Topalian SL, Hodi FS, Brahmer JR, Gettinger SN, Smith DC, McDermott DF, et al. Safety, activity, and immune correlates of anti-PD-1 antibody in cancer. N Engl J Med. 2012;366:2443-54.

2. Snyder A, Makarov V, Merghoub T, Yuan J, Zaretsky JM, Desrichard A, et al. Genetic basis for clinical response to CTLA-4 blockade in melanoma. N Engl J Med. 2014;371:2189-99. 
3. Le DT, Uram JN, Wang H, Bartlett BR, Kemberling H, Eyring AD, et al. PD-1 blockade in tumors with mismatch-repair deficiency. N Engl J Med. 2015;372:2509-20.

4. Hodi FS, O'Day SJ, McDermott DF, Weber RW, Sosman JA, Haanen JB, et al. Improved survival with ipilimumab in patients with metastatic melanoma. N Engl J Med. 2010;363:711-23.

5. Ansell SM, Lesokhin AM, Borrello I, Halwani A, Scott EC, Gutierrez M, et al. PD-1 blockade with nivolumab in relapsed or refractory Hodgkin's lymphoma. N Engl J Med. 2015;372:311-9.

6. Powles T, Eder JP, Fine GD, Braiteh FS, Loriot Y, Cruz C, et al. MPDL3280A (anti-PD-L1) treatment leads to clinical activity in metastatic bladder cancer. Nature. 2014;515:558-62.

7. Ferris RL, Blumenschein G Jr, Fayette J, Guigay J, Colevas AD, Licitra L, et al. Nivolumab for recurrent squamous-cell carcinoma of the head and neck. N Engl J Med. 2016;375:1856-67.

8. McDermott DF, Sosman JA, Sznol M, Massard C, Gordon MS, Hamid O, et al. Atezolizumab, an anti-programmed deathligand 1 antibody, in metastatic renal cell carcinoma: long-term safety, clinical activity, and immune correlates from a phase la study. J Clin Oncol. 2016;34:833-42.

9. Sharma P, Hu-Lieskovan S, Wargo JA, Ribas A. Primary, adaptive, and acquired resistance to cancer immunotherapy. Cell. 2017; 168:707-23.

10. Rizvi NA, Hellmann MD, Snyder A, Kvistborg P, Makarov V, Havel JJ, et al. Cancer immunology. Mutational landscape determines sensitivity to PD-1 blockade in non-small cell lung cancer. Science. 2015;348:124-8.

11. Van Allen EM, Miao D, Schilling B, Shukla SA, Blank C, Zimmer L, et al. Genomic correlates of response to CTLA-4 blockade in metastatic melanoma. Science. 2015;350:207-11.

12. Gao J, Shi LZ, Zhao H, Chen J, Xiong L, He Q, et al. Loss of IFN- $\gamma$ pathway genes in tumor cells as a mechanism of resistance to anti-CTLA-4 therapy. Cell. 2016;167:397-404.e9.

13. Manguso RT, Pope HW, Zimmer MD, Brown FD, Yates KB, Miller BC, et al. In vivo CRISPR screening identifies Ptpn2 as a cancer immunotherapy target. Nature. 2017;547:413-8.

14. Patel SJ, Sanjana NE, Kishton RJ, Eidizadeh A, Vodnala SK, Cam M, et al. Identification of essential genes for cancer immunotherapy. Nature. 2017;548:537-42.

15. Benci JL, Xu B, Qiu Y, Wu TJ, Dada H, Twyman-Saint Victor C, et al. Tumor interferon signaling regulates a multigenic resistance program to immune checkpoint blockade. Cell. 2016;167:1540-54.e12.

16. Zaretsky JM, Garcia-Diaz A, Shin DS, Escuin-Ordinas H, Hugo W, Hu-Lieskovan S, et al. Mutations associated with acquired resistance to PD-1 blockade in melanoma [Internet]. N Engl J Med. 2016:819-29 Available from: https://doi.org/ 10.1056/nejmoa1604958.

17. Rodig SJ, Gusenleitner D, Jackson DG, Gjini E, Giobbie-Hurder A, Jin C, et al. MHC proteins confer differential sensitivity to CTLA-4 and PD-1 blockade in untreated metastatic melanoma. Sci Transl Med. 2018;10 Available from: https://doi. org/10.1126/scitransImed.aar3342.

18. Sumimoto H, Imabayashi F, Iwata T, Kawakami Y. The BRAF-MAPK signaling pathway is essential for cancer-immune evasion in human melanoma cells. J Exp Med. 2006;203:1651-6.

19. Spranger S, Bao R, Gajewski TF. Melanoma-intrinsic $\beta$-catenin signalling prevents anti-tumour immunity. Nature. 2015; 523:231-5.

20. Hugo W, Zaretsky JM, Sun L, Song C, Moreno BH, Hu-Lieskovan S, et al. Genomic and transcriptomic features of response to anti-PD-1 therapy in metastatic melanoma. Cell. 2017;168:542.

21. Peng W, Chen JQ, Liu C, Malu S, Creasy C, Tetzlaff MT, et al. Loss of PTEN promotes resistance to T cell-mediated immunotherapy. Cancer Discov Am Assoc Cancer Res. 2016;6:202-16.

22. Pauken KE, Sammons MA, Odorizzi PM, Manne S, Godec J, Khan O, et al. Epigenetic stability of exhausted T cells limits durability of reinvigoration by PD-1 blockade. Science. 2016;354:1160-5.

23. Sen DR, Kaminski J, Barnitz RA, Kurachi M, Gerdemann U, Yates KB, et al. The epigenetic landscape of T cell exhaustion. Science. 2016;354:1165-9.

24. Yang $W$, Bai $Y$, Xiong $Y$, Zhang J, Chen S, Zheng $X$, et al. Potentiating the antitumour response of $C D 8(+) T$ cells by modulating cholesterol metabolism. Nature. 2016;531:651-5.

25. Zhou P, Shaffer DR, Alvarez Arias DA, Nakazaki Y, Pos W, Torres AJ, et al. In vivo discovery of immunotherapy targets in the tumour microenvironment. Nature. 2014;506:52-7.

26. Thommen DS, Koelzer VH, Herzig P, Roller A, Trefny M, Dimeloe S, et al. A transcriptionally and functionally distinct PD-1 CD8 T cell pool with predictive potential in non-small-cell lung cancer treated with PD-1 blockade. Nat Med. 2018;24:994-1004.

27. Tang H, Wang Y, Chlewicki LK, Zhang Y, Guo J, Liang W, et al. Facilitating T cell infiltration in tumor microenvironment overcomes resistance to PD-L1 blockade. Cancer Cell. 2016;30:500.

28. Cabrita R, Lauss M, Sanna A, Donia M, Skaarup Larsen M, Mitra S, et al. Tertiary lymphoid structures improve immunotherapy and survival in melanoma. Nature. 2020;577:561-5.

29. Helmink BA, Reddy SM, Gao J, Zhang S, Basar R, Thakur R, et al. B cells and tertiary lymphoid structures promote immunotherapy response. Nature. 2020;577:549-55.

30. Petitprez F, de Reyniès A, Keung EZ, Chen TW-W, Sun C-M, Calderaro J, et al. B cells are associated with survival and immunotherapy response in sarcoma. Nature. 2020;577:556-60.

31. De Henau O, Rausch M, Winkler D, Campesato LF, Liu C, Cymerman DH, et al. Overcoming resistance to checkpoint blockade therapy by targeting PI3KY in myeloid cells. Nature. 2016;539:443-7.

32. Kaneda MM, Messer KS, Ralainirina N, Li H, Leem CJ, Gorjestani S, et al. PI3KY is a molecular switch that controls immune suppression. Nature. 2016;539:437-42.

33. Spranger S, Dai D, Horton B, Gajewski TF. Tumor-Residing Batf3 Dendritic Cells Are Required for Effector T Cell Trafficking and Adoptive T Cell Therapy. Cancer Cell. 2017;31:711-23.e4.

34. Barry KC, Hsu J, Broz ML, Cueto FJ, Binnewies M, Combes AJ, et al. A natural killer-dendritic cell axis defines checkpoint therapy-responsive tumor microenvironments. Nat Med. 2018;24:1178-91.

35. Mariathasan S, Turley SJ, Nickles D, Castiglioni A, Yuen K, Wang Y, et al. TGF $\beta$ attenuates tumour response to PD-L1 blockade by contributing to exclusion of T cells. Nature. 2018;554:544 Nature Publishing Group.

36. Sivan A, Corrales L, Hubert N, Williams JB, Aquino-Michaels K, Earley ZM, et al. Commensal Bifidobacterium promotes antitumor immunity and facilitates anti-PD-L1 efficacy. Science. 2015;350:1084-9. 
37. Gopalakrishnan V, Spencer CN, Nezi L, Reuben A, Andrews MC, Karpinets TV, et al. Gut microbiome modulates response to anti-PD-1 immunotherapy in melanoma patients. Science. 2018;359:97-103.

38. Roy S, Trinchieri G. Microbiota: a key orchestrator of cancer therapy. Nat Rev Cancer. 2017;17:271-85.

39. Gajewski TF, Schreiber H, Fu Y-X. Innate and adaptive immune cells in the tumor microenvironment [Internet]. Nat Immunol. 2013:1014-22 Available from: https://doi.org/10.1038/ni.2703.

40. Joyce JA, Fearon DT. T cell exclusion, immune privilege, and the tumor microenvironment. Science. 2015;348:74-80

41. Jiang P, Gu S, Pan D, Fu J, Sahu A, Hu X, et al. Signatures of T cell dysfunction and exclusion predict cancer immunotherapy response. Nat Med. 2018;24:1550-8.

42. Havel JJ, Chowell D, Chan TA. The evolving landscape of biomarkers for checkpoint inhibitor immunotherapy. Nat Rev Cancer. 2019;19:133-50.

43. Lu R, Neff NF, Quake SR, Weissman IL. Tracking single hematopoietic stem cells in vivo using high-throughput sequencing in conjunction with viral genetic barcoding. Nat Biotechnol. 2011;29:928-33.

44. Pei W, Feyerabend TB, Rössler J, Wang X, Postrach D, Busch K, et al. Polylox barcoding reveals haematopoietic stem cell fates realized in vivo. Nature. 2017;548:456-60.

45. Naik SH, Perié L, Swart E, Gerlach C, van Rooij N, de Boer RJ, et al. Diverse and heritable lineage imprinting of early haematopoietic progenitors. Nature. 2013;496:229-32.

46. van Heijst JWJ, Gerlach C, Swart E, Sie D, Nunes-Alves C, Kerkhoven RM, et al. Recruitment of antigen-specific CD8+ T cells in response to infection is markedly efficient. Science. 2009;325:1265-9.

47. Gerlach C, Rohr JC, Perié L, van Rooij N, van Heijst JWJ, Velds A, et al. Heterogeneous differentiation patterns of individual CD8+ T cells. Science. 2013;340:635-9.

48. Reeves MQ, Kandyba E, Harris S, Del Rosario R, Balmain A. Multicolour lineage tracing reveals clonal dynamics of squamous carcinoma evolution from initiation to metastasis. Nat Cell Biol. 2018;20:699-709.

49. Nguyen LV, Pellacani D, Lefort S, Kannan N, Osako T, Makarem M, et al. Barcoding reveals complex clonal dynamics of de novo transformed human mammary cells. Nature. 2015;528:267-71.

50. Wagenblast E, Soto M, Gutiérrez-Ángel S, Hartl CA, Gable AL, Maceli AR, et al. A model of breast cancer heterogeneity reveals vascular mimicry as a driver of metastasis. Nature. 2015;520:358-62.

51. Bhang H-EC, Ruddy DA, Krishnamurthy Radhakrishna V, Caushi JX, Zhao R, Hims MM, et al. Studying clonal dynamics in response to cancer therapy using high-complexity barcoding. Nat Med. 2015;21:440-8.

52. Ben-David U, Siranosian B, Ha G, Tang H, Oren Y, Hinohara K, et al. Genetic and transcriptional evolution alters cancer cell line drug response. Nature. 2018;560:325-30.

53. Gide TN, Quek C, Menzies AM, Tasker AT, Shang P, Holst J, et al. Distinct immune cell populations define response to anti-PD-1 monotherapy and anti-PD-1/anti-CTLA-4 combined therapy. Cancer Cell. 2019;35:238-55.e6.

54. Riaz N, Havel JJ, Makarov V, Desrichard A, Urba WJ, Sims JS, et al. Tumor and microenvironment evolution during immunotherapy with nivolumab [Internet]. Cell. 2017:934-49.e16 Available from: https://doi.org/10.1016/j.cell.2017. 09.028.

55. Snyder A, Nathanson T, Funt SA, Ahuja A, Buros Novik J, Hellmann MD, et al. Contribution of systemic and somatic factors to clinical response and resistance to PD-L1 blockade in urothelial cancer: an exploratory multi-omic analysis. PLoS Med. 2017;14:e1002309.

56. Llosa NJ, Luber B, Siegel N, Awan AH, Oke T, Zhu Q, et al. Immunopathologic stratification of colorectal cancer for checkpoint blockade immunotherapy. Cancer Immunol Res. 2019;7:1574-9.

57. Pan D, Kobayashi A, Jiang P, de Andrade LF, Tay RE, Luoma AM. et al. A major chromatin regulator determines resistance of tumor cells to T cell-mediated killing [Internet]. Science. 2018:770-5 Available from: https://doi.org/ 10.1126/science.aao1710.

58. Kim K, Skora AD, Li Z, Liu Q, Tam AJ, Blosser RL, et al. Eradication of metastatic mouse cancers resistant to immune checkpoint blockade by suppression of myeloid-derived cells. Proc Natl Acad Sci U S A. 2014;111:11774-9.

59. Tumeh PC, Harview CL, Yearley JH, Shintaku IP, Taylor EJM, Robert L, et al. PD-1 blockade induces responses by inhibiting adaptive immune resistance. Nature. 2014;515:568-71.

60. Li B, Li T, Pignon J-C, Wang B, Wang J, Shukla SA, et al. Landscape of tumor-infiltrating T cell repertoire of human cancers. Nat Genet. 2016;48:725-32.

61. Shin DS, Zaretsky JM, Escuin-Ordinas H, Garcia-Diaz A, Hu-Lieskovan S, Kalbasi A, et al. Primary resistance to PD-1 blockade mediated by JAK1/2 mutations. Cancer Discov. 2017;7:188-201.

62. Wang S, Sun H, Ma J, Zang C, Wang C, Wang J, et al. Target analysis by integration of transcriptome and ChIP-seq data with BETA. Nat Protoc. 2013;8:2502-15.

63. Li S, Wan C, Zheng R, Fan J, Dong X, Meyer CA, et al. Cistrome-GO: a web server for functional enrichment analysis of transcription factor ChIP-seq peaks [Internet]. Nucleic Acids Res. 2019; Available from: https://doi.org/ 10.1093/nar/gkz332.

64. Subramanian A, Tamayo P, Mootha VK, Mukherjee S, Ebert BL, Gillette MA, et al. Gene set enrichment analysis: a knowledge-based approach for interpreting genome-wide expression profiles. Proc Natl Acad Sci U S A. 2005;102:15545-50.

65. Fujii T, Horiguchi K, Sunaga H, Moriwaki Y, Misawa H, Kasahara T, et al. SLURP-1, an endogenous a7 nicotinic acetylcholine receptor allosteric ligand, is expressed in CD205(+) dendritic cells in human tonsils and potentiates lymphocytic cholinergic activity. J Neuroimmunol. 2014;267:43-9.

66. Segovia M, Russo S, Jeldres M, Mahmoud YD, Perez V, Duhalde M, et al. Targeting TMEM176B enhances antitumor immunity and augments the efficacy of immune checkpoint blockers by unleashing inflammasome activation. Cancer Cell. 2019;35:767-81.e6.

67. Mei S, Qin Q, Wu Q, Sun H, Zheng R, Zang C, et al. Cistrome Data Browser: a data portal for ChIP-Seq and chromatin accessibility data in human and mouse. Nucleic Acids Res. 2017:45:D658-62.

68. Zheng R, Wan C, Mei S, Qin Q, Wu Q, Sun H, et al. Cistrome Data Browser: expanded datasets and new tools for gene regulatory analysis. Nucleic Acids Res. 2019;47:D729-35.

69. LISA [Internet]. [cited 2019 Jul 21]. Available from: http://lisa.cistrome.org/. 
70. Scheinman RI, Cogswell PC, Lofquist AK, Baldwin AS Jr. Role of transcriptional activation of I kappa B alpha in mediation of immunosuppression by glucocorticoids. Science. 1995;270:283-6.

71. Auphan N, DiDonato JA, Rosette C, Helmberg A, Karin M. Immunosuppression by glucocorticoids: inhibition of NFkappa B activity through induction of I kappa B synthesis. Science. 1995;270:286-90.

72. Cain DW, Cidlowski JA. Immune regulation by glucocorticoids. Nat Rev Immunol. 2017;17:233-47.

73. Soccio RE, Chen ER, Rajapurkar SR, Safabakhsh P, Marinis JM, Dispirito JR, et al. Genetic variation determines PPARY function and anti-diabetic drug response in vivo. Cell. 2015;162:33-44.

74. Stringer-Reasor EM, Baker GM, Skor MN, Kocherginsky M, Lengyel E, Fleming GF, et al. Glucocorticoid receptor activation inhibits chemotherapy-induced cell death in high-grade serous ovarian carcinoma. Gynecol Oncol. 2015;138:656-62.

75. Skor MN, Wonder EL, Kocherginsky M, Goyal A, Hall BA, Cai Y, et al. Glucocorticoid receptor antagonism as a novel therapy for triple-negative breast cancer. Clin Cancer Res. 2013;19:6163-72.

76. Arora VK, Schenkein E, Murali R, Subudhi SK, Wongvipat J, Balbas MD, et al. Glucocorticoid receptor confers resistance to antiandrogens by bypassing androgen receptor blockade. Cell. 2013;155:1309-22.

77. Fensterl V, Sen GC. Interferon-induced Ifit proteins: their role in viral pathogenesis. J Virol. 2015;89:2462-8.

78. Vance RE, Kraft JR, Altman JD, Jensen PE, Raulet DH. Mouse CD94/NKG2A is a natural killer cell receptor for the nonclassical major histocompatibility complex (MHC) class I molecule Qa-1b [Internet]. J Exp Med. 1998:1841-8 Available from: https://doi.org/10.1084/jem.188.10.1841.

79. van Montfoort N, Borst L, Korrer MJ, Sluijter M, Marijt KA, Santegoets SJ, et al. NKG2A blockade potentiates CD8 T cell immunity induced by cancer vaccines. Cell. 2018;175:1744 55.e15.

80. Benci JL, Johnson LR, Choa R, Xu Y, Qiu J, Zhou Z, et al. Opposing functions of interferon coordinate adaptive and innate immune responses to cancer immune checkpoint blockade. Cell. 2019;178:933-48.e14.

81. Williams MJ, Werner B, Heide T, Curtis C, Barnes CP, Sottoriva A, et al. Quantification of subclonal selection in cancer from bulk sequencing data. Nat Genet. 2018;50:895-903.

82. Nathanson T, Ahuja A, Rubinsteyn A, Aksoy BA, Hellmann MD, Miao D, et al. Somatic mutations and neoepitope homology in melanomas treated with CTLA-4 blockade. Cancer Immunol Res. 2017;5:84-91.

83. Leisch F. FlexMix: a general framework for finite mixture models and latent class regression inR [Internet]. J Stat Softw. 2004; Available from: https://doi.org/10.18637/jss.v011.08.

84. Miao D, Margolis CA, Gao W, Voss MH, Li W, Martini DJ, et al. Genomic correlates of response to immune checkpoint therapies in clear cell renal cell carcinoma. Science. 2018;359:801-6.

85. Rooney MS, Shukla SA, Wu CJ, Getz G, Hacohen N. Molecular and genetic properties of tumors associated with local immune cytolytic activity. Cell. 2015;160:48-61.

86. Arya SK, Wong-Staal F, Gallo RC. Dexamethasone-mediated inhibition of human T cell growth factor and gammainterferon messenger RNA. J Immunol. 1984;133:273-6.

87. Obradović MMS, Hamelin B, Manevski N, Couto JP, Sethi A, Coissieux M-M, et al. Glucocorticoids promote breast cancer metastasis. Nature. 2019;567:540-4.

88. Weichselbaum RR, Ishwaran H, Yoon T, Nuyten DSA, Baker SW, Khodarev N, et al. An interferon-related gene signature for DNA damage resistance is a predictive marker for chemotherapy and radiation for breast cancer. Proc Natl Acad Sci U S A. 2008;105:18490-5.

89. Minn AJ. Interferons and the immunogenic effects of cancer therapy [Internet]. Trends Immunol. 2015:725-37 Available from: https://doi.org/10.1016/j.it.2015.09.007.

90. Post AEM, Smid M, Nagelkerke A, Martens JWM, Bussink J, Sweep FCGJ, et al. Interferon-stimulated genes are involved in cross-resistance to radiotherapy in tamoxifen-resistant breast cancer. Clin Cancer Res. 2018;24:3397-408.

91. Pitt JM, Vétizou M, Daillère R, Roberti MP, Yamazaki T, Routy B, et al. Resistance mechanisms to immune-checkpoint blockade in cancer: tumor-intrinsic and -extrinsic factors. Immunity. 2016;44:1255-69.

92. Ishizuka JJ, Manguso RT, Cheruiyot CK, Bi K, Panda A, Iracheta-Vellve A, et al. Loss of ADAR1 in tumours overcomes resistance to immune checkpoint blockade. Nature. 2019;565:43-8.

93. Kearney CJ, Vervoort SJ, Hogg SJ, Ramsbottom KM, Freeman AJ, Lalaoui N, et al. Tumor immune evasion arises through loss of TNF sensitivity. Sci Immunol. 2018;3 Available from: https://doi.org/10.1126/sciimmunol.aar3451.

94. Bunse L, Pusch S, Bunse T, Sahm F, Sanghvi K, Friedrich M, et al. Suppression of antitumor T cell immunity by the oncometabolite (R)-2-hydroxyglutarate. Nat Med. 2018;24:1192-203.

95. Constantinides MG, Link VM, Tamoutounour S, Wong AC, Perez-Chaparro PJ, Han S-J, et al. MAIT cells are imprinted by the microbiota in early life and promote tissue repair. Science. 2019;366 Available from: https://doi. org/10.1126/science.aax6624

96. Legoux F, Bellet D, Daviaud C, El Morr Y, Darbois A, Niort K, et al. Microbial metabolites control the thymic development of mucosal-associated invariant T cells. Science. 2019;366:494-9.

97. Unsworth A, Anderson R, Haynes N, Britt K. OMIP-032: two multi-color immunophenotyping panels for assessing the innate and adaptive immune cells in the mouse mammary gland. Cytometry A. 2016;89:527-30.

98. Dobin A, Davis CA, Schlesinger F, Drenkow J, Zaleski C, Jha S, et al. STAR: ultrafast universal RNA-seq aligner. Bioinformatics. 2013;29:15-21.

99. Li B, Dewey CN. RSEM: accurate transcript quantification from RNA-Seq data with or without a reference genome. BMC Bioinformatics. 2011;12:323.

100. Love Ml, Huber W, Anders S. Moderated estimation of fold change and dispersion for RNA-seq data with DESeq2. Genome Biol. 2014;15:550.

101. Koboldt DC, Chen $K$, Wylie T, Larson DE, McLellan MD, Mardis ER, et al. VarScan: variant detection in massively parallel sequencing of individual and pooled samples. Bioinformatics. 2009;25:2283-5.

102. Hu X, Zhang J, Wang J, Fu J, Li T, Zheng X, et al. Landscape of B cell immunity and related immune evasion in human cancers. Nat Genet. 2019:51:560-7. 
103. Gu S, Wang X, Hu X, Jiang P, Li Z, Traugh N, et al. Clonal tracing reveals diverse patterns of response to immune checkpoint blockade. GSE139476. Gene Expression Omnibus. https://www.ncbi.nlm.nih.gov/geo/query/acc.cgi?acc= GSE139476 (2020).

104. Gu S, Wang X, Hu X, Jiang P, Li Z, Traugh N, et al. Clonal tracing reveals diverse patterns of response to immune checkpoint blockade. Github. https://github.com/Shengqing-Stan-Gu/2020_Clone_Tracing_paper (2020).

105. Gu S, Wang X, Hu X, Jiang P, Li Z, Traugh N, et al. Clonal tracing reveals diverse patterns of response to immune checkpoint blockade. Zenodo https://doi.org/10.5281/zenodo.4016933 (2020).

\section{Publisher's Note}

Springer Nature remains neutral with regard to jurisdictional claims in published maps and institutional affiliations.

Ready to submit your research? Choose BMC and benefit from:

- fast, convenient online submission

- thorough peer review by experienced researchers in your field

- rapid publication on acceptance

- support for research data, including large and complex data types

- gold Open Access which fosters wider collaboration and increased citations

- maximum visibility for your research: over $100 \mathrm{M}$ website views per year

At $\mathrm{BMC}$, research is always in progress.

Learn more biomedcentral.com/submissions 